\title{
Quantum Cohomology via Vicious and Osculating Walkers
}

\author{
CHRISTIAN KORFF \\ School of Mathematics and Statistics, University of Glasgow, 15 University Gardens, \\ G128QW Glasgow, Scotland, UK. e-mail: christian.korff@glasgow.ac.uk
}

Received: 8 October 2013 / Accepted: 4 February 2014

Published online: 6 March 2014 - (C) The Author(s) 2014. This article is published with open access at Springerlink.com

\begin{abstract}
We relate the counting of rational curves intersecting Schubert varieties of the Grassmannian to the counting of certain non-intersecting lattice paths on the cylinder, so-called vicious and osculating walkers. These lattice paths form exactly solvable statistical mechanics models and are obtained from solutions to the Yang-Baxter equation. The eigenvectors of the transfer matrices of these models yield the idempotents of the Verlinde algebra of the gauged $\hat{\mathfrak{u}}(n)_{k}$-WZNW model. The latter is known to be closely related to the small quantum cohomology ring of the Grassmannian. We establish further that the partition functions of the vicious and osculating walker model are given in terms of Postnikov's toric Schur functions and can be interpreted as generating functions for GromovWitten invariants. We reveal an underlying quantum group structure in terms of YangBaxter algebras and use it to give a generating formula for toric Schur functions in terms of divided difference operators which appear in known representations of the nil-Hecke algebra.
\end{abstract}

Mathematics Subject Classification (2010). 14N35, 05E05, 05A15, 05A19, 82B23.

Keywords. Gromov-Witten invariants, quantum cohomology, enumerative combinatorics, exactly solvable models, Bethe ansatz.

\section{Introduction}

Let $\mathrm{Gr}_{n, n+k}$ be the Grassmannian of $n$-planes in $\mathbb{C}^{n+k}$ and consider its small quantum cohomology ring $q H^{*}\left(\mathrm{Gr}_{n, n+k}\right)$. The latter has the following presentation [36]

$$
q H^{*}\left(\mathrm{Gr}_{n, n+k}\right) \cong \mathbb{Z}[q]\left[e_{1}, \ldots, e_{n}, h_{1}, \ldots, h_{k}\right] / \mathcal{I}
$$

where the two-sided ideal $\mathcal{I}$ is generated by the coefficients of the following polynomial in the auxiliary variable $x$,

$$
\left(\sum_{i=0}^{n} e_{i} x^{i}\right)\left(\sum_{j=0}^{k} h_{j} x^{j}\right)=1+(-1)^{n} q x^{N} .
$$


Denoting by $(n, k)$ the set of all partitions whose Young diagram fits into the $n \times k$ bounding box, a vector space basis of $q H^{*}\left(\mathrm{Gr}_{n, n+k}\right)$ is given by the finite set $\left\{s_{\lambda}\right\}_{\lambda \in(n, k)}$ of Schubert classes $s_{\lambda}=\operatorname{det}\left(h_{\lambda_{i}-i+j}\right)_{1 \leq i, j \leq n}=\operatorname{det}\left(e_{\lambda_{i}^{\prime}-i+j}\right)_{1 \leq i, j \leq k}$, where $\lambda^{\prime}$ is the conjugate partition of $\lambda$. Note that this definition includes the special cases $s_{(r)}=h_{r}$ and $s_{\left(1^{r}\right)}=e_{r}$.

\subsection{QUANTUM KOSTKA NUMBERS}

Quantum Kostka numbers were originally introduced in [5] as the coefficients in the following product expansion in (1.1),

$$
s_{\mu} * s_{\lambda_{1}} * \cdots * s_{\lambda_{r}}=\sum_{d \geq 0, v \in(n, k)} q^{d} s_{v} K_{v / d / \mu, \lambda}
$$

where $\mu \in(n, k)$ and $\lambda=\left(\lambda_{1}, \ldots, \lambda_{r}\right)$ are some non-negative integers $\leq k$. As explained in $[5,33]$ the quantum Kostka numbers count certain cylindric skew tableaux, a combinatorial notion first introduced by Gessel and Krattenthaler [18]. Analogously, one can define conjugate quantum Kostka numbers by considering the product expansion

$$
s_{\mu} * s_{\left(1^{\lambda_{1}}\right)} * \cdots * s_{\left(1^{\lambda_{r}}\right)}=\sum_{d \geq 0, v \in(n, k)} q^{d} s_{\nu} K_{v^{\prime} / d / \mu^{\prime}, \lambda}
$$

where $0 \leq \lambda_{i} \leq n$. Due to level-rank duality, $q H^{*}\left(\mathrm{Gr}_{n, n+k}\right) \cong q H^{*}\left(\mathrm{Gr}_{k, n+k}\right)$, one has $K_{v^{\prime} / d / \mu^{\prime}, \lambda}=K_{v / d / \mu, \lambda}$. Nevertheless, both quantum product expansions give rise to different combinatorics, so we will consider them separately.

Exploiting the quantum Giambelli formula [4], $s_{\lambda}=\operatorname{det}\left(s_{\lambda_{i}-i+j}\right)_{1 \leq i, j \leq n}$ with $\lambda \in$ $(n, k)$, one can then compute products between arbitrary Schubert classes

$$
s_{\mu} * s_{\lambda}=\sum_{d \geq 0, v \in(n, k)} q^{d} C_{\lambda \mu}^{\nu, d} s_{\nu}
$$

where the expansion coefficients are the 3-point, genus 0 Gromov-Witten invariants. The latter count rational curves of degree $d=(|\lambda|+|\mu|-|\nu|) / N$ intersecting three Schubert varieties in general position; see, e.g. $[2,4,5,10,11,16]$ for details.

\subsection{TORIC SCHUR POLYNOMIALS AND FROBENIUS STRUCTURES}

Quantum cohomology had its origin in mathematical physics and appeared first in works of Gepner [17], Intriligator [23], Vafa [37] and Witten [38] in connection with the fusion ring $\mathcal{F}_{n, k}$ of the gauged $\widehat{\mathfrak{u}}(n)_{k}$ Wess-Zumino-Novikov-Witten (WZNW) model. It has apparently been proved in the no longer publicly available work [1] that $\mathcal{F}_{n, k} \cong q H^{*}\left(\mathrm{Gr}_{n, n+k}\right) /\langle q-1\rangle . \mathcal{F}_{n, k}^{\mathbb{C}}=\mathcal{F}_{n, k} \otimes_{\mathbb{Z}} \mathbb{C}$ is referred to as Verlinde algebra in the literature. 
Denote by $\lambda^{\vee}$ the partition obtained by taking the complement of the Young diagram of $\lambda$ in the $n \times k$ bounding box and recall the intersection pairing of Schubert classes, $\eta\left(s_{\lambda}, s_{\mu}\right):=\int_{\operatorname{Gr}_{n, n+k}} s_{\lambda} \cdot s_{\mu}=\delta_{\lambda \vee} \mu$.

PROPOSITION 1.1. $\mathcal{F}_{n, k}^{\mathbb{C}}$ with bilinear form $\eta$ is a commutative Frobenius algebra and its coproduct is given by

$$
\Delta_{n, k} s_{\nu}=\sum_{d \geq 0, \mu \in(n, k)} q^{d} s_{\nu / d / \mu} \otimes s_{\mu}, \quad s_{\nu / d / \mu}:=\sum_{\lambda \in(n, k)} C_{\lambda \mu}^{v, d} s_{\lambda} .
$$

Setting $e_{r}=\sum_{1 \leq i_{1}<\cdots<i_{r} \leq n} x_{i_{1}} \cdots x_{i_{r}}$ and $h_{r}=\sum_{1 \leq i_{1} \leq \cdots \leq i_{r} \leq n} x_{i_{1}} \cdots x_{i_{r}}$ where $x=$ $\left(x_{1}, \ldots, x_{n}\right)$ are some commuting indeterminates the Schubert classes can be identified with Schur functions. In light of (1.6) it is then natural to consider so-called toric Schur functions [33]

$$
s_{\nu / d / \mu}(x)=\sum_{\lambda \in(n, k)} C_{\lambda \mu}^{\nu, d} s_{\lambda}(x)=\sum_{\lambda \in(n, k)} K_{\nu / d / \mu, \lambda} m_{\lambda}(x) .
$$

The last identity can be seen as a combinatorial definition of $s_{\nu / d / \mu}$ in terms of monomial symmetric functions; it is the weighted sum over all toric skew tableaux, which is the subset of the cylindric skew tableaux having at most $k$ boxes in each row. This generalises the notion of an ordinary skew Schur function, $s_{\nu / \mu}=$ $\sum_{\lambda} K_{\nu / \mu, \lambda} m_{\lambda}$, where the sum is over all skew (semi-standard) tableaux and $K_{\nu / \mu, \lambda}$ is the ordinary Kostka number; see, e.g. [29]. Since for vanishing degree $d$ the Gromov-Witten invariants equal the Littlewood-Richardson coefficients, one has $s_{\nu / 0 / \mu}=s_{\nu / \mu}$ when $d=0$. In the case of infinitely many variables one obtains cylindric Schur functions which have been investigated in [18,31] and in [28] as special case of affine Stanley symmetric functions; see also [7] for a formulation of a random process on cylindric partitions. For a generalisation of cylindric or toric Schur functions to special cases of Macdonald functions in the context of the $\widehat{\mathfrak{s u}}(n)_{k}$ fusion ring, see [27].

\subsection{EXACTLY SOLVABLE LATTICE MODELS: VICIOUS AND OSCULATING WALKERS}

In this article, we identify the toric Schur polynomial (1.7) with the partition function of exactly solvable lattice models in statistical mechanics [3], the lock-step vicious and osculating walker models which have appeared in connection with problems such as percolation in physical systems [12] and the counting of alternating sign matrices [8]. Both models can be formulated in terms of special nonintersecting paths on a square lattice which in the present context we choose to have dimensions $n \times(n+k)$ and $k \times(n+k)$; see Figure 1 for examples. Fixing start and end positions of the walkers in terms of partitions $\mu, v \in(n, k)$ and identifying the left with the right lattice edge, we show that there is a bijection between toric tableaux of shape $\nu / d / \mu$ and non-intersecting paths of the mentioned mod- 


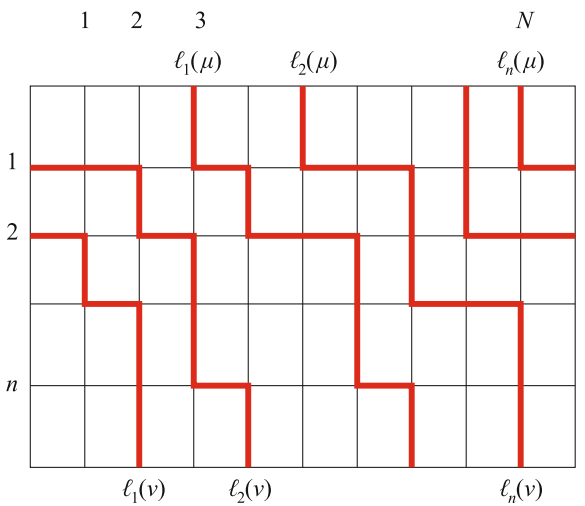

vicious walkers

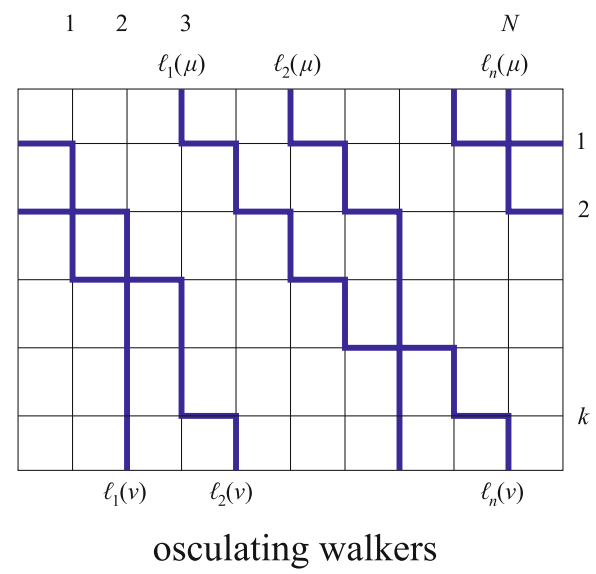

Figure 1. Examples of vicious and osculating walks on a square lattice with $N=n+k=4+5=$ 9. The paths of two osculating walkers can touch but they do not intersect.

els on the cylinder. The degree $d \in \mathbb{Z}_{\geq 0}$ is the number of walkers crossing the boundary, where the horizontal strip is glued together to obtain the cylinder. The weight $\lambda$ of a toric tableau fixes the number of horizontal path edges in each lattice row.

Denote by $H=\left(H_{v, \mu}\right)$ and $E=\left(E_{v, \mu}\right)$ the transfer matrices whose elements are the partition functions of a single lattice row for the vicious and osculating walker models on the cylinder with fixed start and end configurations $\mu, v \in(n, k)$. Then the matrix elements of the powers $H^{n}$ and $E^{k}$ give the partition functions for the lattices with $n$ and $k$ rows mentioned above.

Given a square $s=\langle i, j\rangle$ in the Young diagram of a partition $\lambda$ denote by $c(s)=$ $j-i$ its content and by $h(s)=\lambda_{i}+\lambda_{j}^{\prime}-i-j+1$ its hook length.

THEOREM 1.2. For fixed start and end points $\mu, v \in(n, k)$, the number of vicious and osculating walker configurations on the cylinder is given by

$$
\left(H^{n}\right)_{\nu, \mu}=\sum_{d, \lambda} K_{v / d / \mu, \lambda}=\left(E^{n}\right)_{\nu^{\prime}, \mu^{\prime}}
$$

or alternatively

$$
\left(H^{n}\right)_{\nu, \mu}=\sum_{d, \lambda} C_{\lambda \mu}^{\nu, d} \prod_{s \in \lambda} \frac{n+c(s)}{h(s)}=\left(E^{n}\right)_{\nu^{\prime}, \mu^{\prime}},
$$

where the sums run over all integers $d \geq 0$ and $\lambda \in(n, k)$ such that $|\lambda|+|\mu|-|\nu|=$ $d(n+k)$.

Note that (1.9) provides a linear system of equations for Gromov-Witten invariants. 
It is not obvious from their definition, but we will show that the row-to-row transfer matrices $E$ and $H$ commute and possess a common eigenbasis $\left\{\hat{\mathfrak{e}}_{\lambda}\right\}_{\lambda \in(n, k)}$, the so-called Bethe vectors. They yield the idempotents of the Verlinde algebra $\mathcal{F}_{n, k}^{\mathbb{C}}$ of the gauged $\hat{\mathfrak{u}}(n)_{k}-\mathrm{WZNW}$ model.

THEOREM 1.3 (idempotents of the Verlinde algebra). Let $V_{n, k}$ be the complex linear span of the Bethe vectors $\left\{\hat{\mathfrak{e}}_{\lambda}\right\}_{\lambda \in(n, k)}$. The generalised matrix algebra $\left(V_{n, k}, \star\right)$ obtained by setting $\hat{\mathfrak{e}}_{\lambda} \star \hat{\mathfrak{e}}_{\mu}=\delta_{\lambda \mu} \hat{\mathfrak{e}}_{\lambda}$ is isomorphic to $\mathcal{F}_{n, k}^{\mathbb{C}}$.

The same basis has been employed in [24, Thm 10.11] to provide an alternative derivation of the presentation (1.1). They are identical with the Bethe vectors of the so-called XX-Heisenberg spin chain [24, Rm 10.3] and, thus, one can identify the ring (1.1) with the conserved quantities or "quantum integrals of motion" of this quantum spin-chain. There are close parallels with recent developments regarding the link between topological field theories and quantum integrable models [32] as well as the quantum cohomology of the cotangent bundles of Grassmann varieties $[19,35]$.

The new result in this article is the connection of the quantum cohomology of the Grassmann varieties themselves with the mentioned statistical lattice models which allows one to relate the counting of lattice paths on the cylinder to Gromov-Witten invariants and to reveal a deeper underlying algebraic structure which we now explain.

\subsection{QUANTUM GROUP STRUCTURES}

The combinatorial connection with the mentioned exactly solvable lattice models is underpinned by an algebraic description known as the quantum inverse scattering method which is the name of a general procedure based on the works of the Faddeev School; see, e.g. [6] and references therein. Here, we show that this method can be applied to the quantum cohomology ring. Our starting point is a solution to the Yang-Baxter equation

$$
R_{12}(x / y) M_{1}(x) M_{2}(y)=M_{2}(y) M_{1}(x) R_{12}(x / y)
$$

where $R(x / y) \in \mathbb{C}(x / y) \otimes \operatorname{End}(V \otimes V)$ with $V$ a two-dimensional complex vector space and

$$
M(x)=\left(\begin{array}{ll}
A(x) & B(x) \\
C(x) & D(x)
\end{array}\right) \in \mathbb{C}[x] \otimes \text { End } V \otimes \text { End } V^{\otimes N}
$$

is the so-called monodromy matrix. The matrix entries of $M(x)$ can be interpreted as vertex-type operators whose commutation relations are encoded in the matrix $R$. The latter generate the so-called Yang-Baxter algebra which has the structure 
of a graded bi-algebra. In particular, one can interpret (1.10), (1.11) as the defining relations of a "quantum group"; this is similar to the construction of a Yangian symmetry on the quantum cohomologies of cotangent bundles of Nakajima varieties in a recent preprint by Maulik and Okounkov [30].

Set $N=n+k$ and for simplicity denote $q H^{*}\left(\mathrm{Gr}_{n, k}\right) \otimes_{\mathbb{Z}} \mathbb{C}$ by $q H_{n, k}^{*}$. Then there exists a vector space isomorphism

$$
V^{\otimes N} \otimes \mathbb{C}\left[q, q^{-1}\right] \stackrel{\sim}{\rightarrow} \bigoplus_{n=0}^{N} q H_{n, N-n}^{*}
$$

which induces maps $A_{r}, D_{r}: q H_{n, k}^{*} \rightarrow q H_{n, k}^{*}$ and $B_{r}, C_{r}: q H_{n, k}^{*} \rightarrow q H_{n \pm 1, k \mp 1}^{*}$ where $A_{r}, B_{r}, C_{r}, D_{r}$ are the coefficients of $x^{r}$ in the vertex-type operators (1.11) and $q H_{0, N}^{*}, q H_{N, 0}^{*} \cong \mathbb{C}$. Exploiting level-rank duality, $\Theta: q H_{n, k}^{*} \stackrel{\sim}{\rightarrow} q H_{k, n}^{*}$, one finds a second, dual solution $M^{\prime}=\Theta \circ M \circ \Theta$ of the Yang-Baxter equation. The following result then links these two Yang-Baxter algebras with the Frobenius algebra $q H_{n, k}^{*}$ via (1.2).

THEOREM 1.4. Setting $H(x)=A(x)+q D(x)$ and $E(x)=A^{\prime}(x)+q D^{\prime}(x)$, the restrictions $\left.E(x)\right|_{q H_{n, k}^{*}}$ and $\left.H(x)\right|_{q H_{n, k}^{*}}$ under (1.12) are of polynomial degree $n$ and $k$, respectively and on each preimage of $q H_{n, k}^{*}$ one has the functional identity

$$
E(-x) H(x)=1+(-1)^{n} q x^{N} .
$$

In particular, the map given by $E_{i}=A_{i}^{\prime}+q D_{i}^{\prime} \mapsto e_{i}$ and $H_{j}=A_{j}+q D_{j} \mapsto h_{j}$ with $i=$ $1, \ldots, n$ and $j=1, \ldots, k$ yields an algebra isomorphism $\mathcal{A}_{n, k} \stackrel{\sim}{\rightarrow} q H_{n, k}^{*}$, where $\mathcal{A}_{n, k} \subset$ $\operatorname{End}\left(q H_{n, k}^{*}\right)$ is the commutative subalgebra generated by the coefficients of the YangBaxter algebra elements $E(x)$ and $H(x)$.

The combinatorial results (1.8) and (1.9) then follow from the fact that one recovers the row-to-row vicious and osculating walker transfer matrices at $x=1$, i.e. $H=H(1)$ and $E=E(1)$. An alternative way to express the relation between the Yang-Baxter algebras and $q H_{n, k}^{*}$ is to use the coproduct of the Frobenius algebra.

PROPOSITION 1.5. Setting as before $H(x)=A(x)+q D(x)$ one has for any $1 \leq n \leq$ $N-1$ that

$$
\left\langle\lambda\left|H\left(y_{n}\right) \cdots H\left(y_{1}\right) B\left(x_{n}\right) \cdots B\left(x_{1}\right)\right| 0\right\rangle=x^{\delta_{n}} \Delta_{n, k} s_{\lambda}(y, x)
$$

where $\langle\lambda|$ denotes the dual basis of the Schubert classes under the isomorphism (1.12), $|0\rangle$ the unique basis vectors in $q H_{0, N}^{*}, \delta_{n}=(n, n-1, \ldots, 1)$ and $\Delta_{n, k}$ is the coproduct (1.6) of $q H_{n, k}^{*}$.

This last results implies in particular that one can use the commutation relations of the Yang-Baxter algebra encoded in (1.10) to compute the toric Schur functions 
(1.7) by moving the $H$-operators past the $B$-operators. We will thus derive the following formula in terms of divided difference operators (also called Demazure or Bernstein-Gelfand-Gelfand operators).

Set $\partial_{i}=\left(1-x_{i+1} / x_{i}\right)^{-1}\left(s_{i}-1\right)$ with $s_{i}$ being the transposition which acts by switching $x_{i}$ with $x_{i+1}$ and define $\nabla_{i}=\partial_{n-1+i} \cdots \partial_{i+1} \partial_{i}$.

COROLLARY 1.6. Choose $y_{i}=x_{i+n}$ in (1.14) and introduce the "generating function"

$$
F_{\lambda}(x ; y)=s_{\lambda}(y) \prod_{i=1}^{n}\left(1+q x_{i}^{N}\right) .
$$

Then we have the following formula in terms of Demazure operators,

$$
\sum_{d \geq 0} q^{d} s_{\lambda / d / \mu}(y)=\left\langle s_{\mu}(x) \| x^{-\delta_{n}} \nabla_{1} \nabla_{2} \cdots \nabla_{n} y^{\delta_{n}} F_{\lambda}(x ; y)\right\rangle
$$

where the notation $\left\langle s_{\mu}(x) \| \cdots\right\rangle$ denotes the coefficient of the Schur function $s_{\mu}(x)$.

We will demonstrate on a simple example how toric Schur functions can be explicitly computed by invoking the last formula in Section 6 .

Another identity for toric Schur functions - which is a direct consequence of the quantum group structure and does not seem to have appeared previously in the literature - is the following sum rule.

COROLLARY 1.7. Let $N=2 n$. Then we have the following identities for toric Schur functions

$$
e_{r}\left(x_{1}^{N}, \ldots, x_{n}^{N}\right)=\sum_{\substack{d+d^{\prime}=r \\ \mu \in(n, n)}}(-1)^{|\lambda|-|\mu|} s_{\lambda^{\prime} / d^{\prime} / \mu^{\prime}}\left(x_{1}, \ldots, x_{n}\right) s_{\mu / d / \lambda}\left(x_{1}, \ldots, x_{n}\right),
$$

where $\lambda$ is any partition in the $n \times n$ square.

This identity is a true "quantum relation" as it becomes trivial for $q=0$, i.e. there exists no analogue of this relation for skew Schur functions where $d, d^{\prime}=0$. Similar identities hold also for $N \notin 2 \mathbb{N}$ but look more complicated. They will be stated in Section 6.

\subsection{OUTLINE OF THE ARTICLE}

In Section 2, we introduce some preliminary combinatorial notions regarding 01words and partitions.

In Section 3, we discuss in detail the vicious and osculating walker models. While these have been introduced in the literature previously, our conventions differ from the usual ones by rotating the lattice $45^{\circ}$ and choosing a special set of 
weights. We also analyse in depth the related Yang-Baxter algebras and show that both models are related via level-rank duality. We derive a matrix functional equation relating the transfer matrices of both models.

Section 4 contains the algebraic Bethe ansatz construction of the idempotents of the Verlinde algebra. As a byproduct of this construction, we obtain novel expressions for Schur functions as matrix elements of the above mentioned Yang-Baxter algebras.

Section 5 states explicit bijections between vicious and osculating walker configurations on the cylinder and toric tableaux, which can be interpreted as a special subset of semi-standard tableau of skew shape. As a corollary, we obtain that Postnikov's toric Schur polynomials are the partition functions of the specialised vicious and osculating walker models. The sum rule (1.9), relating Gromov-Witten invariants to the counting of vicious and osculating walker configurations on the cylinder, is then an immediate consequence.

We will conclude with stating the proofs of the various identities for toric Schur functions arising from the quantum group structure.

\section{Preliminaries}

Throughout this article we consider non-negative integers $N, n, k \in \mathbb{Z}_{\geq 0}$ such that $N=n+k$ and set $I:=\{1, \ldots, N\}$. Let $V=\mathbb{C} v_{0} \oplus \mathbb{C} v_{1}$ and denote by $V^{*}$ its dual. Consider the tensor product $V^{\otimes N}$. We identify the standard basis $\mathcal{B}=\left\{v_{w_{1}} \otimes \cdots \otimes\right.$ $\left.v_{w_{N}}: w_{i}=0,1\right\} \subset V^{\otimes N}$ with the set of 01-words of length $N, W=\left\{w=w_{1} w_{2} \ldots w_{N}\right.$ : $\left.w_{i}=0,1\right\}$, via the map

$$
w \mapsto|w\rangle:=v_{w_{1}} \otimes \cdots \otimes v_{w_{N}} .
$$

For convenience, we are employing the Dirac notation and denote by $\langle w|$ the dual basis with $\langle w \mid \tilde{w}\rangle=\prod_{i=1}^{N} \delta_{w_{i}, \tilde{w}_{i}}$. Furthermore, we shall denote by $W_{n}=\{w \in W:|w|=$ $\left.\sum_{i=1}^{N} w_{i}=n\right\}$ the subset of all 01-words with $n$ 1-letters, by $\mathcal{B}_{n} \subset V^{\otimes N}$ its image under the above map (2.1) and by $V_{n} \subset V^{\otimes N}$ the subspace spanned by the corresponding basis elements in $\mathcal{B}_{n}$. As (2.1) is a bijection, we can also introduce the inverse map whose image we denote by $w(b)$ with $b \in \mathcal{B}_{n}$.

There are alternative descriptions of the elements in $\mathcal{B}_{n}$ which will be useful for our discussion. Namely, consider the set of partitions $\lambda$ whose Young diagram fits into a bounding box of height $n$ and width $k$; we shall denote it by $(n, k)$. Define a bijection $(n, k) \rightarrow W_{n}$ via

$$
\lambda \mapsto w(\lambda)=0 \cdots 0 \ell_{\ell_{1}}^{10 \cdots 0} \operatorname{l}_{\ell_{n}} 0 \cdots 0, \quad \ell_{i}(\lambda)=\lambda_{n+1-i}+i,
$$

where $\ell(\lambda)=\left(\ell_{1}, \ldots, \ell_{n}\right)$ with $1 \leq \ell_{1}<\cdots<\ell_{n} \leq N$ denote the positions of 1-letters in $w(\lambda)$ from left to right. We assume the latter to be periodic, that is we set $\ell_{i+n}(\lambda)=\ell_{i}(\lambda)+N$. We shall denote the image of the inverse of the map (2.2) by $\lambda(w)$ and by $|\lambda\rangle$ the corresponding ket vector in $\mathcal{B}_{n}$. Note that the correspondence 


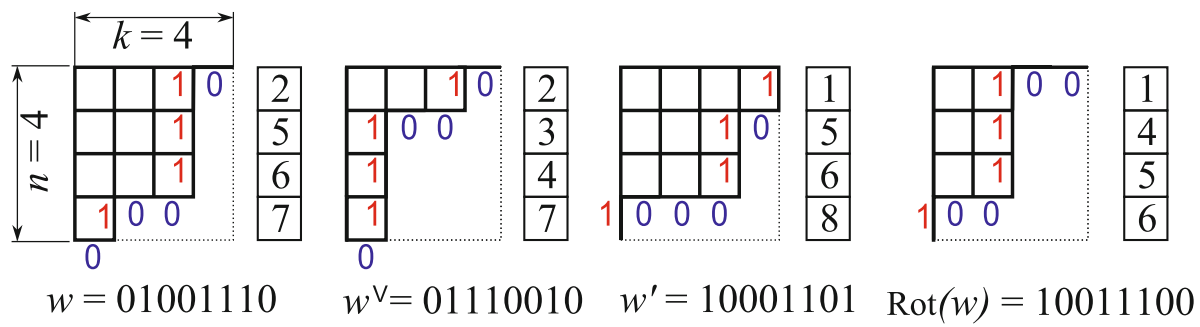

Figure 2. 01-words, partitions and single column tableaux. Depicted are the various transformations of 01-word defined in the text for $N=2 n=2 k=8$.

(2.2) can be easily understood graphically: the Young diagram of the partition $\lambda$ traces out a path in the $n \times k$ rectangle which is encoded in $w$. Starting from the left bottom corner in the $n \times k$ rectangle go one box right for each letter 0 and one box up for each letter 1; see Figure 2 for an example. For later purposes, we introduce the notation

$$
n_{\ell}(\lambda)=\sum_{i=1}^{\ell} w_{i}(\lambda)
$$

for $\ell \in I$ and set $n_{\ell+N}(\lambda)=n_{\ell}(\lambda)+n$ for any $\ell \in \mathbb{Z}$.

Exploiting the bijection (2.2) there are two operations on 01 -words which are induced by taking the complement of $\lambda \in(n, k)$ in the bounding box, $\lambda \mapsto \lambda^{\vee}:=$ $\left(k-\lambda_{n}, \ldots, k-\lambda_{2}, k-\lambda_{1}\right)$, and by considering the conjugate partition $\lambda^{\prime} \in(k, n)$. The corresponding 01-words $w\left(\lambda^{\vee}\right), w\left(\lambda^{\prime}\right)$ are obtained from $w(\lambda)$ via the maps

$$
w \mapsto w^{\vee}:=w_{N} \ldots w_{2} w_{1} \quad \text { and } \quad w \mapsto w^{\prime}=\left(1-w_{N}\right) \cdots\left(1-w_{2}\right)\left(1-w_{1}\right) \text {, }
$$

respectively. Note that these maps yield bijections $W_{n} \rightarrow W_{n}$ and $W_{n} \rightarrow W_{k}$. We will also make use of the combined map $w \mapsto w^{\#}:=\left(w^{\vee}\right)^{\prime}=\left(w^{\prime}\right)^{\vee}$ which is simply the exchange of 0 and 1-letters.

There is one additional map Rot: $W_{n} \rightarrow W_{n}$ which we require for our discussion: set $w \mapsto \operatorname{Rot}(w):=w_{2} w_{3} \ldots w_{N} w_{1}$ which translates via (2.2) to the action

$$
\operatorname{Rot}(\lambda):=\left\{\begin{array}{cc}
\left(\lambda_{1}-1, \ldots, \lambda_{n}-1\right), & \lambda_{n}>0 \\
\left(k, \lambda_{1}, \ldots, \lambda_{n-1}\right), & \text { else }
\end{array} .\right.
$$

Exploiting the last expression one then derives the following formula with $|\lambda|=$ $\sum_{i} \lambda_{i}$

$$
\left|\operatorname{Rot}^{\ell}(\lambda)\right|=|\lambda|-\ell n+n_{\ell}(\lambda) N .
$$

Note that obviously we have $\operatorname{Rot}^{N}(\lambda)=\lambda$. For obvious reasons we will refer to Rot as the rotation operator. The maps (2.4) and (2.5) are significant for our discussion as they constitute symmetries of Gromov-Witten invariants. 


\section{Vicious and Osculating Walkers}

We recall the definition of the lock step vicious walker model originally introduced by Fisher [12] and show that this statistical mechanics model with the correct choice of weights and boundary conditions is closely related to the small quantum cohomology ring of the Grassmannian: its partition function can be interpreted as generating function of 3-point, genus zero Gromov-Witten invariants.

There is another statistical model introduced by Brak [8], called osculating walkers, ${ }^{1}$ which in our setting turns out to be dual or complementary to the vicious walker model. Namely, we will show that the transfer matrices of the vicious and osculating walker models are given in terms of analogues of complete and elementary symmetric functions in certain noncommutative variables: the generators of the nil affine Temperley-Lieb algebra.

\subsection{VICIOUS WALKERS: VERTEX AND LATTICE CONFIGURATIONS}

We start with the 5-vertex formulation of the vicious walker model. Fix two integers $N>0$ and $0 \leq n \leq N$ and consider the square lattice

$$
\mathbb{L}:=\left\{\langle i, j\rangle \in \mathbb{Z}^{2} \mid 0 \leq i \leq n+1,0 \leq j \leq N+1\right\} .
$$

Denote by $\mathbb{E}=\left\{\left(p, p^{\prime}\right) \in \mathbb{L}^{2}: p_{1}+1=p_{1}^{\prime}, p_{2}=p_{2}^{\prime}\right.$ or $\left.p_{1}=p_{1}^{\prime}, p_{2}+1=p_{2}^{\prime}\right\}$ the set of horizontal and vertical edges.

DEFINITION 3.1. A lattice configuration $\mathcal{C}: \mathbb{E} \rightarrow\{0,1\}$ is an assignment of values 0 or 1 to the lattice edges.

The weight of a configuration $\mathcal{C}$ is defined as the product over its vertex weights,

$$
\operatorname{wt}(\mathcal{C})=\prod_{(i, j) \in \mathbb{L}} \operatorname{wt}\left(\mathrm{v}_{i, j}\right) \in \mathbb{Z}\left[x_{1}, \ldots, x_{n}\right],
$$

where $\mathrm{v}_{i, j}$ denotes the vertex obtained by intersecting the $i$ th horizontal lattice line with the $j$ th vertical one. That is, a vertex configuration is a 4-tuple $\mathrm{v}_{i, j}=$ $(a, b, c, d)$ where, respectively, $a, b, c, d=0,1$ are the values of the $\mathrm{W}, \mathrm{N}, \mathrm{E}, \mathrm{S}$ edges at the lattice point $\langle i, j\rangle$. There are five allowed vertex configurations which are depicted in Figure 3 together with their weights. All other vertex configurations are forbidden, i.e. they have weight zero. Some of the nonzero weights are given in terms of a set of commutative indeterminates $\left(x_{1}, \ldots, x_{n}\right)$, one for each row.

Connecting the 1-letters in each vertex configuration as shown in Figure 3, it is easy to see that each lattice configuration corresponds to a configuration of

${ }^{1}$ We note that Brak's model is a six-vertex model, i.e. has different Boltzmann weights from the one discussed here and in particular has one more allowed vertex configuration. However, the crucial vertex configuration with two paths approaching each other arbitarily close is also present here and we therefore adopt his nomenclature; see Figure 4. 


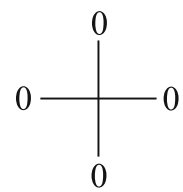

weight: 1

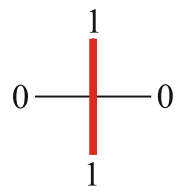

1

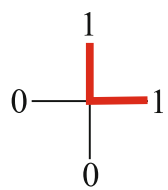

1

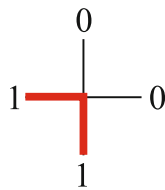

$x_{i}$

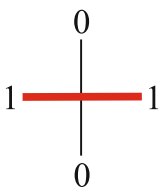

$x_{i}$

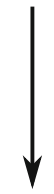

time

Figure 3. The five allowed vertex configurations and their corresponding vicious walk sections.

$n$ non-intersecting paths, where a path $\gamma=\left(p_{1}, \ldots, p_{l}\right)$ is a sequence of points $p_{r}=\left(i_{r}, j_{r}\right) \in \mathbb{L}$ such that either $p_{r+1}=\left(i_{r}+1, j_{r}\right)$ or $\left(i_{r}, j_{r}+1\right)$, i.e. a connected sequence of horizontal and vertical edges as depicted in Figure 8.

\subsection{TRANSFER MATRIX AND NIL TEMPERLEY-LIEB POLYNOMIALS}

Define $\sigma^{-}=\left(\begin{array}{ll}0 & 1 \\ 0 & 0\end{array}\right), \sigma^{+}=\left(\begin{array}{ll}0 & 0 \\ 1 & 0\end{array}\right), \sigma^{z}=\left(\begin{array}{ll}1 & 0 \\ 0 & -1\end{array}\right)$ to be the Pauli matrices acting on $V \cong \mathbb{C}^{2}$ via $\sigma^{-} v_{1}=v_{0}, \sigma^{+} v_{0}=v_{1}$ and $\sigma^{z} v_{\alpha}=(-1)^{\alpha} v_{\alpha}, \alpha=0,1$. We now interpret the possible vertex configuration in the $i^{\text {th }}$ row and $j^{\text {th }}$ column as a map $L\left(x_{i}\right)$ : $V_{i}\left(x_{i}\right) \otimes V_{j} \rightarrow V_{i}\left(x_{i}\right) \otimes V_{j}$ with $V_{i}\left(x_{i}\right)=V_{i} \otimes \mathbb{C}\left(x_{i}\right)$ and $V_{i} \cong V_{j} \cong V$ for all $\langle i, j\rangle \in \mathbb{L}$. We will therefore drop the row and column labels and, in addition, often suppress the dependence on the indeterminate $x_{i}$ in the notation. Thus, the values of the horizontal edges label the basis vectors in $V_{i}$ while the values of the vertical edges label the basis vectors in $V_{j}$. The mapping is from the NW to the SE direction through the vertex. That is, label with $a, b, c, d=0,1$ the values of the edges in Figure 3 in clockwise direction starting from the $\mathrm{W}$ edge. Interpret the corresponding weight $L_{c d}^{a b}=\operatorname{wt}\left(\mathrm{v}_{i, j}\right)$ as the matrix element of the map $L$, where we set $L_{c d}^{a b}=0$ whenever the vertex configuration is not allowed. We then obtain

$$
L\left(x_{i}\right) v_{a} \otimes v_{b}=\sum_{c, d=0,1} L_{c d}^{a b}\left(x_{i}\right) v_{c} \otimes v_{d}=x_{i}^{a}\left[v_{0} \otimes\left(\sigma^{+}\right)^{a} v_{b}+v_{1} \otimes \sigma^{-}\left(\sigma^{+}\right)^{a} v_{b}\right]
$$

which can be rewritten in the basis $\left\{v_{0} \otimes v_{0}, v_{0} \otimes v_{1}, v_{1} \otimes v_{0}, v_{1} \otimes v_{1}\right\}$ as

$$
L\left(x_{i}\right)=\left(\begin{array}{cccc}
1 & 0 & 0 & 0 \\
0 & 1 & x_{i} & 0 \\
0 & 1 & x_{i} & 0 \\
0 & 0 & 0 & 0
\end{array}\right)
$$

PROPOSITION 3.2. The 5-vertex L-matrix satisfies the Yang-Baxter equation,

$$
R_{12}(x, y) L_{13}(x) L_{23}(y)=L_{23}(y) L_{13}(x) R_{12}(x, y),
$$


where the matrix $R$ is given by

$$
R(x, y)=\left(\begin{array}{cccc}
1 & 0 & 0 & 0 \\
0 & 0 & 1 & 0 \\
0 & y / x & 1-y / x & 0 \\
0 & 0 & 0 & y / x
\end{array}\right)
$$

Proof. A straightforward computation.

Note that the matrix $R(x, y)$ is non-singular for generic $x, y$, since det $R(x, y)=$ $-y^{2} / x^{2}$. The solution $L$ to the Yang-Baxter equation can be used to define an algebra in $\operatorname{End}(V)^{\otimes N} \cong \operatorname{End}\left(V^{\otimes N}\right)$, called the Yang-Baxter algebra which plays a central role in the quantum inverse scattering method; see, e.g. [6] for a textbook and references therein. In fact, the Yang-Baxter algebra comes naturally equipped with a coproduct. First rewrite the $L$-matrix in the form

$$
L(x)=\left(\begin{array}{cc}
1 & x \sigma^{+} \\
\sigma^{-} & x \sigma^{-} \sigma^{+}
\end{array}\right)
$$

where the matrix elements are polynomials in the indeterminate $x$ with coefficients in End $V$. The following is a known result how to introduce a bi-algebra structure on solutions of the Yang-Baxter equation; we therefore omit the proof.

PROPOSITION 3.3. Define implicitly a coproduct $\Delta: \operatorname{End}(V) \rightarrow \operatorname{End}(V) \otimes \operatorname{End}(V)$ by setting $\Delta L(x):=L_{13}(x) L_{12}(x)$ and a co-unit $\varepsilon: \operatorname{End}(V) \rightarrow \mathbb{C}$ by $\varepsilon(L(x))=\left(\begin{array}{ll}1 & 0 \\ 0 & 1\end{array}\right)$. Here the maps $\varepsilon, \Delta$ act on the coefficients when expanding with respect to the spectral variable $x$. The set of solutions of (3.5) equipped with $\Delta, \varepsilon$ forms a bialgebra, so in particular $\Delta L$ is again a solution of (3.5).

Note that we do not have a Hopf algebra structure as the $L$-operator is not invertible.

Repeatedly applying $\Delta$ and the isomorphism $\operatorname{End}(V) \otimes \operatorname{End}(W) \cong \operatorname{End}(V \otimes W)$, one is led to consider the so-called monodromy matrix in $\operatorname{End}(V(x)) \otimes \operatorname{End}\left(V^{\otimes N}\right)$

$$
T(x)=L_{0 N}(x) \cdots L_{02}(x) L_{01}(x)=\left(\begin{array}{ll}
A(x) & B(x) \\
C(x) & D(x)
\end{array}\right) .
$$

From the Yang-Baxter equation one then deduces - among others - the following commutation relations for the entries of the monodromy matrix,

$$
\begin{aligned}
& A(x) A(y)=A(y) A(x), D(x) D(y)=D(y) D(x), \\
& B(x) B(y)=\frac{y}{x} B(y) B(x), C(x) C(y)=\frac{x}{y} C(y) C(x)
\end{aligned}
$$


and

$$
\begin{aligned}
& x B(x) A(y)=x B(y) A(x)+(x-y) A(y) B(x), \\
& y B(x) D(y)+(x-y) D(x) B(y)=y B(y) D(x) .
\end{aligned}
$$

We now describe these commutation relations in terms of divided difference operators. This will allow us in a subsequent section to derive the generating function (1.15) for toric Schur functions and formula (1.16) mentioned in the introduction. We will also use these relations below to construct eigenvectors of the vicious walker transfer matrix.

Consider the polynomial ring $\mathcal{R}_{m}=\mathbb{Z}\left[x_{1}, \ldots, x_{m}\right]$ where the $x_{i}$ 's are some commuting indeterminates. There is a natural action of the symmetric group $S_{m}$ on $\mathcal{R}_{m}$ by permuting the $x_{i}$ 's where we denote by $\left\{s_{i}\right\}_{i=1}^{m-1}$ the elementary transpositions which exchange $x_{i}$ and $x_{i+1}$. Introduce the difference operators $\partial_{i}=(1-$ $\left.x_{i+1} / x_{i}\right)^{-1}\left(s_{i}-1\right)$ for $i=1, \ldots, m$. Despite first appearance, the latter map polynomials into polynomials: because of linearity it suffices to consider the following action on a monomial

$$
\partial_{i} x_{i}^{a} x_{i+1}^{b}=\left\{\begin{array}{cc}
\sum_{r=0}^{b-a-1} x_{i}^{b-r} x_{i+1}^{a+r}, & a<b \\
0, & a=b . \\
-\sum_{r=0}^{a-b-1} x_{i}^{a-r} x_{i+1}^{b+r}, & a>b
\end{array}\right.
$$

The difference operators yield a representation of the nil Hecke algebra $\mathcal{H}_{m}(0)$, that is they obey the relations

$$
\partial_{i}^{2}=-\partial_{i} \quad \text { and } \quad \partial_{i} \partial_{i+1} \partial_{i}=\partial_{i+1} \partial_{i} \partial_{i+1}
$$

Remark 3.4. The action (3.11) of the difference operators $\partial_{i}$ is the familiar action of the Hecke algebra $\mathcal{H}_{m}(\mathfrak{q})$ on the ring of polynomials via Demazure or BernsteinGelfand-Gelfand operators in the limit $\mathfrak{q} \rightarrow 0$. In this limit, the algebra $\mathcal{H}_{m}(0)$ is known as the nil-Hecke algebra.

We now have the following simple but important lemma.

LEMMA 3.5. Let $f \in \mathcal{R}_{m} \otimes V^{\otimes N}$ and suppose $s_{i} f=f$ for all $i=1, \ldots, m-1$. Then we have the commutation relations

$$
\begin{aligned}
& A\left(x_{i+1}\right) B\left(x_{i}\right) f=\partial_{i} B\left(x_{i+1}\right) A\left(x_{i}\right) f \\
& D\left(x_{i+1}\right) B\left(x_{i}\right) f=-\partial_{i} B\left(x_{i+1}\right) A\left(x_{i}\right) f \\
& C\left(x_{i+1}\right) B\left(x_{i}\right) f=\partial_{i} D\left(x_{i+1}\right) A\left(x_{i}\right) f .
\end{aligned}
$$

Proof. This is a direct computation using (3.5), (3.6) and the definition (3.8). 
To describe the action of the matrix elements $A, B, C, D$ in combinatorial terms we now relate them to a particular representation of the affine nil Temperley-Lieb algebra. The latter is the unital, associative algebra generated by $\left\{u_{1}, \ldots, u_{N}\right\}$ and relations

$$
\begin{aligned}
u_{i}^{2} & =u_{i} u_{i+1} u_{i}=u_{i+1} u_{i} u_{i+1}=0, \quad i \in \mathbb{Z}_{N} \\
u_{i} u_{j} & =u_{j} u_{i}, \quad|i-j| \quad \bmod N>1 .
\end{aligned}
$$

We will refer to the subalgebra generated by $\left\{u_{1}, \ldots, u_{N-1}\right\}$ as the finite nil Temperley-Lieb algebra. Note that the latter is a quotient of the nil Hecke algebra.

PROPOSITION 3.6 hopping operators. The map

$$
u_{i} \mapsto f_{i}:=\sigma_{i+1}^{+} \sigma_{i}^{-}, \quad i=1, \ldots, N-1
$$

yields a faithful representation of the finite nil Temperley-Lieb algebra over $V_{n}$. If we further set

$$
u_{N} \mapsto f_{N}:=q \sigma_{1}^{+} \sigma_{N}^{-}
$$

we obtain a faithful representation of the affine nil Temperley-Lieb algebra over $\mathbb{C}\left[q, q^{-1}\right] \otimes V_{n}$

Remark 3.7. In [24] a free fermion description of the small quantum cohomology ring was presented. The relationship between the current description of the affine nil Temperley-Lieb algebra and that in loc. cit. is given via the following formulae

$$
\psi_{i}=\sigma_{i}^{-} \prod_{j<i} \sigma_{j}^{z} \quad \text { and } \quad \psi_{i}^{*}=\sigma_{i}^{+} \prod_{j<i} \sigma_{j}^{z}
$$

In particular, one easily verifies that $f_{i}=\psi_{i+1}^{*} \psi_{i}=\sigma_{i+1}^{+} \sigma_{i}^{-}$with

$$
\psi_{i+N}=-q^{-1} \psi_{i} \prod_{j=1}^{N} \sigma_{j}^{z} \quad \text { and } \quad \psi_{i+N}^{*}=-q \psi_{i}^{*} \prod_{j=1}^{N} \sigma_{j}^{z} .
$$

The last proposition is then an obvious reformulation of [24, Prop 9.1] and we therefore omit the proof.

The action (3.16) suggest to introduce the following quasi-periodic boundary conditions, $\sigma_{i+N}^{ \pm}=-q^{ \pm 1} \sigma_{i}^{ \pm}$and $\sigma_{i+N}^{z}=\sigma_{i}^{z}$. We also introduce the adjoint endomorphisms $f_{i}^{*}=\sigma_{i+1}^{-} \sigma_{i}^{+}$and $f_{N}^{*}=q^{*} \sigma_{1}^{-} \sigma_{N}^{+}$, where $q^{*}=q^{-1}$. For ease of notation, we will henceforth simply write $V_{n, q}:=\mathbb{C}\left[q, q^{-1}\right] \otimes V_{n}$ and $V_{q}^{\otimes N}:=\mathbb{C}\left[q, q^{-1}\right] \otimes V^{\otimes N}$.

One easily deduces the following identities which we state without proof; compare with [24, Section 8.2 and Lemma 9.3]. 
LEMMA 3.8. Denote by $\Theta: b \mapsto b^{\prime}, \vee: b \mapsto b^{\vee}$ and $\operatorname{Rot}: b \mapsto \operatorname{Rot} b$ the endomorphisms $V^{\otimes N} \rightarrow V^{\otimes N}$ induced by the maps in (2.4) and (2.5). Then

$$
\vee \circ f_{i}=f_{N-i}^{*} \circ \vee, \quad \Theta \circ f_{i}=f_{N-i} \circ \Theta, \quad \operatorname{Rot} \circ f_{i+1}=f_{i} \circ \text { Rot, }
$$

where all indices are understood modulo $N$.

PROPOSITION 3.9. We have the following expressions for the Yang-Baxter algebra in terms of the $f_{i}$ 's:

$$
\begin{aligned}
& A(x)=\left(1+x f_{N-1}\right) \cdots\left(1+x f_{1}\right), \\
& B(x)=x A(x) \sigma_{1}^{+}, \quad C(x)=\sigma_{N}^{-} A(x), \quad D(x)=x \quad \sigma_{N}^{-} A(x) \sigma_{1}^{+} .
\end{aligned}
$$

Proof. From the definition of the monodromy matrix one easily derives the expression

$$
T_{b, a}(x)=\sum_{\alpha} x^{|\alpha|+a}\left(\sigma_{N}^{-}\right)^{b} f_{N-1}^{\alpha_{N-1}} \cdots f_{1}^{\alpha_{1}}\left(\sigma_{1}^{+}\right)^{a},
$$

where the sum runs over all compositions $\alpha=\left(\alpha_{1}, \ldots, \alpha_{N-1}\right)$ with $\alpha_{i}=0,1$. The assertion is now immediate.

The action of the operators $A_{r}$ in $V_{n} \subset V^{\otimes N}$ is easily described using the wellknown bijections between 01-words and partitions explained in Section 2.

LEMMA 3.10 (horizontal strips). Let $\mu \in(n, k)$, and $A(x)=\sum_{r \geq 0} x^{r} A_{r}$. Then the polynomials

$$
A_{r}=\sum_{\alpha \vdash r} f_{N-1}^{\alpha_{N-1}} \cdots f_{1}^{\alpha_{1}}
$$

act on the basis vector $|\mu\rangle$ by adding all possible horizontal $r$-strips to the Young diagram of $\mu$ such that the result $\lambda$ lies within the $n \times k$ bounding box, $A_{r}|\mu\rangle=$ $\sum_{\lambda-\mu=(r)}|\lambda\rangle$.

Remark 3.11. Polynomials in noncommutative variables such as (3.20) have been considered in e.g. [13] and their affine extensions in [33] and [24,25]. Here the new aspect is that we show that they are part of the Yang-Baxter algebra of the vicious walker model.

Proof. Using the bijection (2.2) one readily verifies that either $f_{i}|\mu\rangle=|\lambda\rangle$, where $\lambda$ is obtained by adding a box in the $(i-n)$ th diagonal of the Young diagram of $\mu$, or if this is not possible $f_{i}|\mu\rangle=0$. Consider now a consecutive string $f_{i+r^{\prime}} \cdots f_{i+1}$ $f_{i}|\mu\rangle=|\lambda\rangle$ with $r^{\prime} \leq r$ and suppose $w_{i}(\mu)=1, w_{j}(\mu)=0$ for $i<j \leq i+r^{\prime}$; otherwise the action is trivial. Then the 1-letter at position $i$ in $w(\mu)$ is moved past $r^{\prime} 0$ letters whose position each decreases by one. Since $\mu_{k+1-j}^{\prime}=\ell_{j}\left(\mu^{\prime}\right)+j$, where $N+$ 
$1-\ell_{k+1-j}\left(\mu^{\prime}\right)$ are the positions of 0-letters in $w(\mu)$, we find $\lambda_{k+1-j}^{\prime}-\mu_{k+1-j}^{\prime}=1$. Thus, $\lambda$ is obtained from $\mu$ by adding a horizontal strip of length $r^{\prime}$. This proves the assertion.

From the last lemma, the action of the remaining Yang-Baxter algebra generators is obtained by observing that $\sigma_{1}^{+}|\lambda\rangle=\left|\lambda_{1}-1, \ldots, \lambda_{n}-1\right\rangle$ if $\lambda_{n}>0$ or $\sigma_{1}^{+}|\lambda\rangle=0$ if $\lambda_{n}=0$. In contrast, $\sigma_{N}^{-}|\lambda\rangle=|\mu\rangle$ if $\mu$ can be obtained by adding a column of maximal height to the Young diagram of $\lambda$ and then subtracting a boundary ribbon of length $N$ starting in the first row. Otherwise, we have $\sigma_{N}^{-}|\lambda\rangle=0$.

LEMMA 3.12. (i) The operator $B_{r}$ acts by adding all possible horizontal strips of length $r-1$ such that the result lies within the $n \times k$ bounding box and removing the leftmost column of the resulting Young diagram afterwards. (ii) In contrast, the operator $C_{r}$ acts by adding all possible horizontal strips of length $r$ and removing the top row afterwards. (iii) Finally, the action of the operator $D_{r}$ is to first add an horizontal r-strip, preserving the height but not the width of the bounding box, and then subtracting a boundary ribbon of length $N$ starting from the first row.

From the commutation relations (3.9) of the Yang-Baxter algebra it now follows that certain polynomials in the noncommutative alphabets $\left\{f_{1}, \ldots, f_{N-1}\right\}$ and $\left\{f_{1}, \ldots, f_{N}\right\}$ commute.

COROLLARY 3.13 (integrability). (i) The finite nil Temperley-Lieb polynomials $A_{r}$ commute pairwise, that is $A_{r} A_{r^{\prime}}=A_{r^{\prime}} A_{r}, \forall r, r^{\prime}=0,1, \ldots, N$.

(ii) Let $H(x)=A(x)+q D(x) \in \operatorname{End} V_{q}^{\otimes N}$ then one has the expansion

$$
H(x)=\sum_{r=0}^{N} x^{r} H_{r}, \quad H_{r}:=\sum_{\alpha \vdash r} q^{\alpha_{N}}\left(\sigma_{N}^{-}\right)^{\alpha_{N}} f_{N-1}^{\alpha_{N-1}} \cdots f_{1}^{\alpha_{1}}\left(\sigma_{1}^{+}\right)^{\alpha_{N}}
$$

Moreover, $H_{r} H_{r^{\prime}}=H_{r^{\prime}} H_{r}$ for all $r, r^{\prime}=0,1, \ldots, N$.

Proof. The first assertion is immediate from the commutation relation (3.9). The operator $H: V_{q}^{\otimes N} \rightarrow V_{q}^{\otimes N}$ is the so-called row-to-row transfer matrix of the vicious walker model on the cylinder, i.e. its matrix elements are the partition functions of one lattice row when imposing quasi-periodic boundary conditions in the horizontal direction of the square lattice. The transfer matrix can be written as the following partial trace,

$$
H(x)=\operatorname{Tr}_{0} q^{\left(\sigma^{+} \sigma^{-}\right)_{0}} L_{0 N}(x) \cdots L_{01}(x),
$$

where the indices indicate in which factors of the tensor product $V(x) \otimes V_{q}^{\otimes N}$ the respective $L$-operators act. (The factors are labelled from left to right starting with $0,1, \ldots, N$.) The additional operator $q^{\sigma^{+} \sigma^{-}}$under the trace invokes quasiperiodic boundary conditions, i.e. the powers of the indeterminate $q$ count how 
many outer horizontal edges are occupied. It is now a consequence of the YangBaxter equation that this model is integrable, i.e. the transfer matrices commute pairwise, $H(x) H(y)=H(y) H(x)$ for any $x, y$. The assertion then follows from the expansion (3.19).

For completeness, we summarise our previous findings on the combinatorial action of the Yang-Baxter algebra (3.18) in the following formula for each $r=$ $0,1, \ldots, N$.

LEMMA 3.14. Let $\mu \in(n, k)$ then

$$
H_{r}|\mu\rangle=\sum_{\lambda-\mu=(r)}|\lambda\rangle+q \sum_{\lambda[1]-\mu=(r)}|\lambda\rangle,
$$

where in the second sum $\lambda[1]$ denotes the partition obtained from $\lambda$ by adding $a$ boundary ribbon of length $N$ starting in the first and ending in the $n^{\text {th }}$ row. For $r>k$, we have $H_{r}|\mu\rangle=0$.

Proof. We postpone a detailed proof to Section 5 where we discuss the bijection between row configurations of the vicious walker model and toric tableaux; see the proof of Proposition 5.8.

\subsection{OSCULATING WALKERS: VERTEX AND LATTICE CONFIGURATIONS}

Define another 5-vertex model but this time on a $k \times N$ lattice with $k=N-n$,

$$
\mathbb{L}^{\prime}:=\left\{\langle i, j\rangle \in \mathbb{Z}^{2} \mid 0 \leq i \leq k+1,0 \leq j \leq N+1\right\}
$$

Denote by $\mathbb{E}^{\prime}$ the set of its horizontal and vertical edges. As before, we define the weight of a lattice configuration $\mathcal{C}: \mathbb{E}^{\prime} \rightarrow\{0,1\}$ as $\mathrm{wt}^{\prime}(\mathcal{C})=\prod_{(i, j) \in \mathbb{L}^{\prime}} \mathrm{wt}^{\prime}\left(\mathrm{v}_{i, j}\right) \in$ $\mathbb{Z}\left[x_{1}, \ldots, x_{k}\right]$, where the allowed vertex configurations and their weights $\mathrm{wt}^{\prime}\left(\mathrm{v}_{i, j}\right)$ are depicted in Figure 4. The weights of the allowed vertex configurations again depend only on the row index of the square lattice via the commutative indeter-

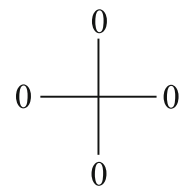

weight: 1

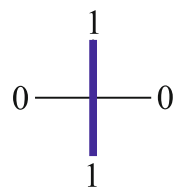

1

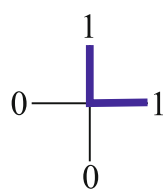

1

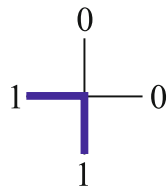

$x_{i}$

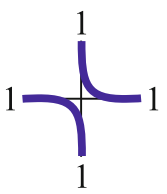

$x_{i}$

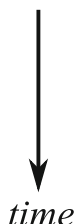

time

Figure 4. The five allowed vertex configurations for the osculating walker model. Note that this model differs from the vicious walker model in the last vertex configuration only. 
minates $\left(x_{1}, \ldots, x_{k}\right)$. The corresponding $L^{\prime}$-matrix reads

$$
L^{\prime} v_{a} \otimes v_{b}=\sum_{c, d=0,1} L_{c d}^{\prime a b} v_{c} \otimes v_{d}=x_{i}^{a}\left[v_{0} \otimes\left(\sigma^{+}\right)^{a} v_{b}+v_{1} \otimes\left(\sigma^{+}\right)^{a} \sigma^{-} v_{b}\right],
$$

which can be rewritten in the basis $\left\{v_{0} \otimes v_{0}, v_{0} \otimes v_{1}, v_{1} \otimes v_{0}, v_{1} \otimes v_{1}\right\}$ as

$$
L^{\prime}\left(x_{i}\right)=\left(\begin{array}{cccc}
1 & 0 & 0 & 0 \\
0 & 1 & x_{i} & 0 \\
0 & 1 & 0 & 0 \\
0 & 0 & 0 & x_{i}
\end{array}\right) .
$$

Via a straightforward computation, which we omit, one arrives at the following result.

PROPOSITION 3.15. We have the identity

$$
R_{12}^{\prime}(x / y) L_{13}^{\prime}(x) L_{23}^{\prime}(y)=L_{23}^{\prime}(y) L_{13}^{\prime}(x) R_{12}^{\prime}(x / y),
$$

where

$$
R^{\prime}(x / y)=\left(\begin{array}{cccc}
1 & 0 & 0 & 0 \\
0 & 1-x / y & x / y & 0 \\
0 & 1 & 0 & 0 \\
0 & 0 & 0 & x / y
\end{array}\right) .
$$

Note that the matrix $R^{\prime}(x / y)$ is non-singular for generic $x, y$.

In complete analogy with our previous discussion of the vicious walker model, we can define also here a monodromy matrix

$$
T^{\prime}(x)=L_{0 N}^{\prime}(x) \cdots L_{02}^{\prime}(x) L_{01}^{\prime}(x)=\left(\begin{array}{ll}
A^{\prime}(x) & B^{\prime}(x) \\
C^{\prime}(x) & D^{\prime}(x)
\end{array}\right)
$$

and Yang-Baxter algebra.

PROPOSITION 3.16. The matrix elements in (3.28) are given by the following expressions in the hopping operators (3.15),

$$
\begin{aligned}
& A^{\prime}(x)=\left(1+x f_{1}\right) \cdots\left(1+x f_{N-1}\right) \\
& B^{\prime}(x)=x \sigma_{1}^{+} A^{\prime}(x), \quad C^{\prime}(x)=A^{\prime}(x) \sigma_{N}^{-}, \quad D^{\prime}(x)=x \quad \sigma_{1}^{+} A^{\prime}(x) \sigma_{N}^{-}
\end{aligned}
$$

and we have the commutation relations $A^{\prime}(x) A^{\prime}(y)=A^{\prime}(y) A^{\prime}(x)$.

Proof. Exploiting (3.25) one derives from the definition (3.28) the expansion

$$
T_{b, a}^{\prime}(x)=\sum_{\alpha} x^{|\alpha|+a}\left(\sigma_{1}^{+}\right)^{a} f_{1}^{\alpha_{1}} \cdots f_{N-1}^{\alpha_{N-1}}\left(\sigma_{N}^{-}\right)^{b},
$$


where the sum runs again over all compositions $\alpha=\left(\alpha_{1}, \ldots, \alpha_{N-1}\right)$ with $\alpha_{i}=0,1$.

Similar like before, one now verifies the following combinatorial action of the Yang-Baxter algebra.

LEMMA 3.17 (vertical strips). Let $A^{\prime}(x)=\sum_{r \geq 0} x^{r} A_{r}^{\prime}$. The polynomials

$$
A_{r}^{\prime}=\sum_{\alpha \vdash r} f_{1}^{\alpha_{1}} \cdots f_{N-1}^{\alpha_{N-1}}
$$

act on $|\mu\rangle$ with $\mu \in(n, k)$ a partition by adding all possible vertical $r$-strips to the Young diagram of $\mu$ such that the resulting diagram lies still within the $n \times k$ bounding box, $A_{r}^{\prime}|\mu\rangle=\sum_{\lambda / \mu=\left(1^{r}\right)}|\lambda\rangle$.

Proof. Similar to the case of vicious walkers we consider $f_{i} f_{i+1} \cdots f_{i+r}|\mu\rangle=|\lambda\rangle$ which is non-trivial only if $w_{i+r^{\prime}+1}(\mu)=0$ and $w_{j}(\mu)=1$ with $i \leq j \leq i+r^{\prime}, r^{\prime} \leq r$. Employing (2.2) we find that $\lambda_{n+1-j}-\mu_{n+1-j}=1$. Thus, $\lambda$ is obtained by adding a vertical strip of height $r$.

We now impose again quasi-periodic boundary conditions in the horizontal direction of the square lattice and introduce the corresponding transfer matrix $E$ : $V_{q}^{\otimes N} \rightarrow V_{q}^{\otimes N}$

$$
E\left(x_{i}\right):=A^{\prime}\left(x_{i}\right)+q D^{\prime}\left(x_{i}\right)=\operatorname{Tr}_{0} q^{\left(\sigma^{+} \sigma^{-}\right)_{0}} L_{0 N}^{\prime}\left(x_{i}\right) \cdots L_{01}^{\prime}\left(x_{i}\right) .
$$

As in the case of the vicious walkers model one now exploits the Yang-Baxter equation to arrive at the following set of commuting polynomials in the $f_{i}$ 's.

COROLLARY 3.18 (integrability). We have the expansion

$$
E(x)=\sum_{r=0}^{N} x^{r} E_{r}, \quad E_{r}=\sum_{\alpha \vdash r} q^{\alpha_{N}}\left(\sigma_{1}^{+}\right)^{\alpha_{N}} f_{1}^{\alpha_{1}} \cdots f_{N-1}^{\alpha_{N-1}}\left(\sigma_{N}^{-}\right)^{\alpha_{N}}
$$

and the $\left\{E_{r}\right\}_{r=0}^{N}$ commute pairwise.

The following lemma shows that vicious and osculating walkers are related via level-rank duality.

LEMMA 3.19 (level-rank duality). Let $\Theta: V_{q}^{\otimes N} \rightarrow V_{q}^{\otimes N}$ be the involution induced by sending each $|w\rangle \in \mathcal{B}$ to $\left|w^{\prime}\right\rangle$; compare with (2.4). Then we have $\Theta \circ H_{r}=E_{r} \circ \Theta$ for all $r=0,1, \ldots, N$. 
Proof. This is an easy consequence of the second relation in (3.17) and the formulae (3.21), (3.34).

An immediate consequence is the following combinatorial action of the $E_{r}$ 's which simply follows from the combinatorial action of the vicious walker transfer matrix discussed previously.

LEMMA 3.20. Let $\mu \in(n, k)$ then

$$
E_{r}|\mu\rangle=\sum_{\lambda-\mu=\left(1^{r}\right)}|\lambda\rangle+q \sum_{\lambda[1]-\mu=\left(1^{r}\right)}|\lambda\rangle,
$$

where in the second sum $\lambda[1]$ again denotes the partition obtained from $\lambda$ by adding $a$ boundary ribbon of length $n+k$ starting in the first and ending in the $n^{\text {th }}$ row. For $r>n$, we have $E_{r}|\mu\rangle=0$.

We can also determine the commutation relations between the Yang-Baxter algebras of the vicious and osculating walker models via a third and final Yang-Baxter relation, which - again - is obtained by a tedious but direct computation which we omit.

PROPOSITION 3.21. We have the additional identity

$$
R_{12}^{\prime \prime}(x / y) L_{1}(x) L_{2}^{\prime}(y)=L_{2}^{\prime}(y) L_{1}(x) R_{12}^{\prime \prime}(x / y)
$$

where $R^{\prime \prime}$ is the singular matrix

$$
R^{\prime \prime}(x / y)=\left(\begin{array}{cccc}
1+x / y & 0 & 0 & 0 \\
0 & 1 & x / y & 0 \\
0 & 1 & x / y & 0 \\
0 & 0 & 0 & 0
\end{array}\right) .
$$

Exploiting this last result one now proves in a similar manner as before that the transfer matrices $H(x)$ and $E(y)$ commute for arbitrary $x, y$.

COROLLARY 3.22. We have the following commutation relations

$$
\begin{aligned}
A(x) A^{\prime}(y) & =A^{\prime}(y) A(x), \\
(x+y) A^{\prime}(y) \sigma_{N}^{-} A(x) & =x \sigma_{N}^{-} A(x) A^{\prime}(y)+y A(x) A^{\prime}(y) \sigma_{N}^{-}, \\
(x+y) A(x) \sigma_{1}^{+} A^{\prime}(y) & =x A(x) A^{\prime}(y) \sigma_{1}^{+}+y \sigma_{1}^{+} A(x) A^{\prime}(y),
\end{aligned}
$$

and

$y A(x) \sigma_{1}^{+} A^{\prime}(y) \sigma_{N}^{-}+x \sigma_{N}^{-} A(x) \sigma_{1}^{+} A^{\prime}(y)=y \sigma_{1}^{+} A^{\prime}(y) \sigma_{N}^{-} A(x)+x A^{\prime}(y) \sigma_{N}^{-} A(x) \sigma_{1}^{+}$.

In particular, finite and affine nil Temperley-Lieb polynomials $H_{r}, E_{r}$ pairwise commute, i.e. $H_{r} E_{r^{\prime}}=E_{r^{\prime}} H_{r}$ for all $r, r^{\prime}=0,1, \ldots, N$. 
We have the following functional relation between the transfer matrices $H, E$ which generalises the known relation of the generating functions for elementary and complete symmetric polynomials in the ring of symmetric functions.

PROPOSITION 3.23. We have the operator identity

$$
H(x) E(-x)=1+q x^{N} \prod_{j=1}^{N} \sigma_{j}^{z} .
$$

Proof. Decompose $V \otimes V$ into $W \oplus W^{\perp}$ with $W=\operatorname{ker} R^{\prime \prime}(-1)$. Namely, $W$ is spanned by $w_{1}=v_{0} \otimes v_{0}, w_{2}=v_{0} \otimes v_{1}+v_{1} \otimes v_{0}, w_{3}=v_{1} \otimes v_{1}$, while $W^{\perp}=\mathbb{C}\left\{w_{4}\right\}$ with $w_{4}=\frac{1}{2}\left(v_{1} \otimes v_{0}-v_{0} \otimes v_{1}\right)$. From the Yang-Baxter equation (3.36) for $y=-x$ one deduces that $L_{13}(x) L_{23}^{\prime}(-x) W \otimes V \subset W \otimes V$. Thus, we can block decompose $L_{13}(x) L_{23}^{\prime}(-x)=\left(\begin{array}{ll}M & * \\ 0 & M^{\prime}\end{array}\right)$ with respect to $W \oplus W^{\perp}$ and one only needs to verify that $M, M^{\prime}$ yield the asserted terms on the right hand side of (3.40) using that

$$
L_{13}(x) L_{23}^{\prime}(-x) w_{1} \otimes v_{0}=w_{1} \otimes v_{0}, \quad L_{13}(x) L_{23}^{\prime}(-x) w_{1} \otimes v_{1}=w_{1} \otimes v_{1}+w_{2} \otimes v_{0}
$$

and

$$
L_{13}(x) L_{23}^{\prime}(-x) w_{2} \otimes v_{0,1}=L_{13}(x) L_{23}^{\prime}(-x) w_{3} \otimes v_{0,1}=0 .
$$

We leave the details of this last step to the reader since it is a simple computation.

COROLLARY 3.24. Let $\mathcal{A}_{n, k} \subset \operatorname{End}\left(V_{n, q}\right)$ be the commutative algebra generated by $\left\{H_{j}\right\}_{j=0}^{k}$ and $\left\{E_{i}\right\}_{i=0}^{n}$. The map $E_{i} \mapsto e_{i}$ and $H_{j} \mapsto h_{j}$ provides a canonical algebra isomorphism $\mathcal{A}_{n, k} \cong q H^{*}\left(\mathrm{Gr}_{n, n+k}\right) \otimes_{\mathbb{Z}} \mathbb{C}$.

Proof. This is clear from our previous results: the combinatorial actions (3.23) and (3.35). In particular, one has that $H_{r} \mid V_{n, q}=0$ for $r>k$ and $E_{r} \mid V_{n, q}=0$ for $r>$ $n$. The Yang-Baxter relations (3.5), (3.26), (3.36) provided us with a proof that the $E_{i}$ 's and $H_{j}$ 's commute among themselves and with each other. Finally, the last result (3.40) gives the desired algebraic dependence expressed in (1.2).

\section{Algebraic Bethe Ansatz and Idempotents}

We show in this section that the eigenvectors of the transfer matrices $H, E$ for the vicious and osculating walker models are the idempotents of the fusion ring of the gauged WZNW model. The eigenbasis of the affine nil Temperley-Lieb polynomials $H_{\lambda}:=H_{\lambda_{1}} H_{\lambda_{2}} \ldots$ and $E_{\lambda}:=E_{\lambda_{1}} E_{\lambda_{2}} \ldots$ with $\lambda_{i}<N$ has been previously constructed in [24, Section 10] using the free fermion formalism mentioned earlier. 
Here, we obtain a new result: we show that the same eigenbasis can be obtained from the Yang-Baxter algebras (3.18), (3.30) of the vicious and osculating walker models by a procedure known as algebraic Bethe ansatz; see, e.g. [6] for a textbook reference. In particular, this construction will furnish us with the vertex-type operator formulae ( 4.3), (4.4) for Schur functions which differ from the known expressions; see, e.g. [29, Chapter I, Section 5, Ex 29] and references therein.

\subsection{BETHE VECTORS}

We assume that $q^{ \pm 1 / N}$ exist and define $\mathfrak{e}_{\lambda}=\mathfrak{e}\left(y_{1}(\lambda), \ldots, y_{n}(\lambda)\right)$ where

$$
\mathfrak{e}\left(y_{1}, \ldots, y_{n}\right):=\sum_{\lambda \in(n, k)} s_{\lambda}\left(y_{1}^{-1}, \ldots, y_{n}^{-1}\right)|\lambda\rangle
$$

and $y_{j}(\lambda)=q^{\frac{1}{N}} \exp \left[\frac{2 \pi i}{N} J_{j}(\lambda)\right]$ with $J_{j}(\lambda)=-\frac{n+1}{2}+\lambda_{j}+j$ and $\lambda \in(n, k)$. We recall from [24, Section 10] the following results.

\section{THEOREM 4.1 (Korff-Stroppel).}

(i) The vectors $\left\{\mathfrak{e}_{\lambda}\right\}_{\lambda \in(n, k)}$ form an orthogonal basis of $V_{n}$, and we have that

$$
\left\|\mathfrak{e}_{\lambda}\right\|^{2}:=\sum_{\mu} s_{\mu}\left(y_{1}, \ldots, y_{n}\right) s_{\mu}\left(y_{1}^{-1}, \ldots, y_{n}^{-1}\right)=\frac{2^{\frac{n(1-n)}{2}} k(n+k)^{k}}{\prod_{i<j} \sin ^{2} \frac{\pi}{n+k}\left(\lambda_{i}-\lambda_{j}+i-j\right)} .
$$

(ii) The basis $\left\{\mathfrak{e}_{\lambda}\right\}_{\lambda \in(n, k)}$ diagonalises the $H_{\mu}$ 's and $E_{\mu}$ 's; one has $H_{\mu} \mathfrak{e}_{\lambda}=h_{\mu}(y(\lambda)) \mathfrak{e}_{\lambda}$ and $E_{\mu} \mathfrak{e}_{\lambda}=e_{\mu}(y(\lambda)) \mathfrak{e}_{\lambda}$ for all compositions $\mu$ with $\mu_{i}<N$.

The following identities which connect the eigenvectors with the Yang-Baxter algebras are new and are not contained in [24, Section 10].

LEMMA 4.2. Let $|0\rangle,|N\rangle$ be the basis vectors in the one-dimensional spaces $V_{0}$ and $V_{N}$. We have the following expansions,

$$
B\left(x_{1}\right) \cdots B\left(x_{n}\right)|0\rangle=x^{\delta_{n}} \sum_{\lambda \in(n, k)} s_{\lambda}(x)|\lambda\rangle
$$

with $\delta_{n}=(n, n-1, \ldots, 1)$ and

$$
C^{\prime}\left(x_{1}\right) \cdots C^{\prime}\left(x_{k}\right)|N\rangle=x^{\rho_{k}} \sum_{\lambda \in(n, k)} s_{\lambda^{\prime}}(x)|\lambda\rangle,
$$

where $\rho_{k}=(k, \ldots, 2,1)$. 

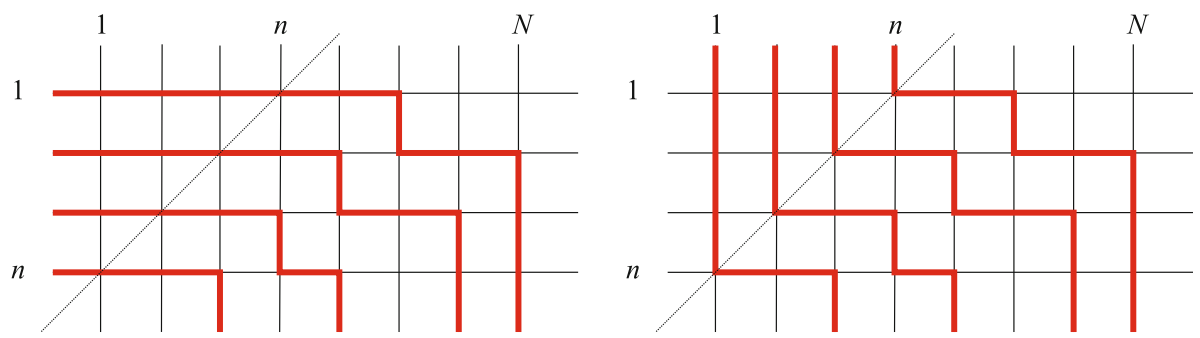

Figure 5. Vicious walker configurations for the $B$-operator (left) and the $A$-operator $($ right $)$.

So, in particular, we have the following identities between matrix elements and Schur functions,

$$
s_{\lambda}\left(x_{1}, \ldots, x_{n}\right)=x^{-\delta}\left\langle\lambda\left|B\left(x_{1}\right) \cdots B\left(x_{n}\right)\right| 0\right\rangle=x^{-\rho_{n}}\left\langle\lambda^{\prime}\left|C^{\prime}\left(x_{1}\right) \cdots C^{\prime}\left(x_{n}\right)\right| N\right\rangle .
$$

Proof. The proof is graphical. Draw a diagonal line across the square lattice as indicated in Figure 5.

Claim 4.3. The vicious walker configurations shown on the right in Figure 5 are in bijection with semi-standard tableaux $T$ of shape $\lambda \in(n, k)$ where $\ell_{i}(\lambda), i=1, \ldots n$ defined in (2.2) are the end positions of the walkers and the corresponding weight of such configurations is $x^{\alpha}$, where $\alpha \in \mathbb{Z}_{\geq 0}^{n}$ is the weight of the tableau.

We postpone the proof of this claim to the next section where we establish a bijection between non-intersecting paths on the cylinder and toric Young tableaux. The claim will then follow as a special case by considering only those vertex weights which lie below the dotted line and noting that the vertices above the line contribute the total weight factor $x_{1}^{n} x_{2}^{n-1} \cdots x_{n}$. Thus, redrawing the lattice paths as indicated in Figure 5 and using the known sum formula $s_{\lambda}(x)=\sum_{|T|=\lambda} x^{T}$ (see, e.g. [29, Chapter I]), we arrive at the desired expression

$$
B\left(x_{n}\right) \cdots B\left(x_{1}\right)|0\rangle=x_{1}^{n} x_{2}^{n-1} \cdots x_{n} \sum_{\lambda \in(n, k)} s_{\lambda}(x)|\lambda\rangle .
$$

The second identity simply uses the fact that $\Theta \sigma_{i}^{+} \Theta=\sigma_{N-i}^{-}$, which is easily verified. Thus, from $\Theta A(x) \Theta=A^{\prime}(x)$ (see Lemma 3.19 for $q=0$ ) and (3.18), (3.30) it follows that $\Theta B(x) \Theta=x C^{\prime}(x)$. Applying the involution $\Theta$ on both sides of the previous identity and swapping $n$ and $k$ afterwards, the second assertion is proved.

PROPOSITION 4.4. Fix $0 \leq n<N$. For each $\lambda \in(n, k)$, the vector $\mathfrak{e}_{\lambda}$ is an eigenvector of $H=A+q D$ and $E=A^{\prime}+q D^{\prime}$ with 


$$
\begin{aligned}
H(x) \mathfrak{e}_{\lambda} & =\left(1+(-1)^{n} q x^{N}\right) \prod_{i=1}^{n} \frac{1}{1-x y_{i}(\lambda)} \mathfrak{e}_{\lambda}, \\
E(x) \mathfrak{e}_{\lambda} & =\prod_{i=1}^{n}\left(1+x y_{i}(\lambda)\right) \mathfrak{e}_{\lambda} .
\end{aligned}
$$

For $n=N$ the eigenvalue equations simplify to $H(x)|N\rangle=|N\rangle$ and $E(x)|N\rangle=(1+$ $\left.q x^{N}\right)|N\rangle$.

Proof. Start with $n=1$. Note that $A(x)|0\rangle=|0\rangle$ and $D(x)|0\rangle=x^{N}|0\rangle$. According to $(3.10)$, we then find that

$$
B(y)|0\rangle=\sum_{r>0} y^{r}|0 \cdots 010 \cdots 0\rangle
$$

is an eigenvector of $H(x)$ with eigenvalue $\left(1+q x^{N}\right) /(1-x / y)$ provided that $y^{N} q=$ 1. This computation generalises to $n>1$, using an induction argument one finds with the help of the equations (3.10),

$$
\begin{aligned}
A(x) B\left(y_{n}\right) \cdots B\left(y_{1}\right)= & B\left(y_{n}\right) \cdots B\left(y_{1}\right) A(x) \prod_{i=1}^{n} \frac{y_{i}}{y_{i}-x} \\
& -\sum_{i=1}^{n} B\left(y_{n}\right) \cdots B(x) \cdots B\left(y_{1}\right) A\left(y_{i}\right) \frac{y_{i}}{y_{i}-x} \prod_{j \neq i} \frac{y_{i} y_{j}}{y_{j}-y_{i}}
\end{aligned}
$$

and

$$
\begin{aligned}
D(x) B\left(y_{n}\right) \cdots B\left(y_{1}\right)= & B\left(y_{n}\right) \cdots B\left(y_{1}\right) D(x) \prod_{i=1}^{n} \frac{y_{i}}{x-y_{i}} \\
& -\sum_{i=1}^{n} B\left(y_{n}\right) \cdots B(x) \cdots B\left(y_{1}\right) D\left(y_{i}\right) \frac{y_{i}}{x-y_{i}} \prod_{j \neq i} \frac{y_{i} y_{j}}{y_{i}-y_{j}},
\end{aligned}
$$

Employing these identities, one deduces that for $\mathfrak{e}(y)$ to be an eigenvector the socalled Bethe roots $y_{i}$ have to satisfy the following set of constraints,

$$
y_{1}^{N}=\cdots=y_{n}^{N}=(-1)^{n-1} q .
$$

The explicit solution to these equations is easily obtained and can be found in [24, Prop 10.4].

To arrive at the eigenvalue equation for $E$ one can either perform a similar computation using the operators $C^{\prime}$ introduced earlier and employing level-rank duality or employ Proposition 3.23.

The statement for $n=N$ is obvious and follows from the definition of $H, E$.

Let $\mathcal{P}$ be the linear operator $V^{\otimes N} \rightarrow V^{\otimes N}$ defined by $\mathcal{P}|\lambda\rangle=\left|\lambda^{\vee}\right\rangle$ and $\mathcal{T}$ the linear operator defined by $\mathcal{T}|\lambda\rangle=|\lambda\rangle$ and $\mathcal{T} q=q^{-1} \mathcal{T}$ for each $\lambda \in(n, k)$ with $N=$ $n+k$. We introduce the operators 


$$
H^{*}(x)=\mathcal{P} \mathcal{T} \quad H(x) \mathcal{P} \mathcal{T} \quad \text { and } \quad E^{*}(x)=\mathcal{P} \mathcal{T} \quad E(x) \mathcal{P} \mathcal{T} .
$$

Note that it follows from the definition that we have the identity $H^{*}(x)=A^{*}(x)+$ $q^{-1} D^{*}(x)$ with

$$
L_{01}(x) L_{02}(x) \cdots L_{0 N}(x)=\left(\begin{array}{ll}
A^{*}(x) & B^{*}(x) \\
C^{*}(x) & D^{*}(x)
\end{array}\right) .
$$

An analogous formula holds for $E^{*}(x)$.

LEMMA 4.5. We have the dual affine Pieri rules

$$
H_{r}^{*}|\mu\rangle=\sum_{\mu-\lambda=(r)}|\lambda\rangle+q^{-1} \sum_{\mu[1]-\lambda=(r)}|\lambda\rangle
$$

and

$$
E_{r}^{*}|\mu\rangle=\sum_{\mu-\lambda=\left(1^{r}\right)}|\lambda\rangle+q^{-1} \sum_{\mu[1]-\lambda=\left(1^{r}\right)}|\lambda\rangle,
$$

where the notation $\mu[1]$ in the second sum in both formulae stands for the partition obtained by adding a boundary rim hook of length $n+k$ to $\mu$.

Proof. Simply note that $\mathcal{P} \sigma_{i}^{+} \sigma_{i+1}^{-}=\sigma_{N-i}^{-} \sigma_{N+1-i}^{+} \mathcal{P}$. The rest is then a straightforward computation which follows along similar lines as in the case of $H_{r}$ and $E_{r}$ using the explicit polynomial expressions in the $f_{i}$ 's given in (3.21), (3.34).

The last result motivates us to define an isomorphism $\left(V_{q}^{\otimes N}\right)^{*} \rightarrow V_{q}^{\otimes N}$ by identifying the dual basis vector $\langle\lambda|$ with $\mathcal{P}|\lambda\rangle=\left|\lambda^{\vee}\right\rangle$, and more generally any vector $v^{*}=\sum_{\lambda} c_{\lambda}(q)\langle\lambda| \in\left(V_{q}^{\otimes N}\right)^{*}$ with $v=\sum_{\lambda} c_{\lambda}\left(q^{-1}\right)\left|\lambda^{\vee}\right\rangle \in V_{q}^{\otimes N}$. In other words, the pairing $\langle v \mid w\rangle$ between the vector space and its dual is given by the bilinear form $\eta(v, w):=(v, \mathcal{P} \mathcal{T} w)$, where $(|\lambda\rangle,|\mu\rangle)=\delta_{\lambda \mu}$.

LEMMA 4.6. The left or dual eigenvectors $\mathfrak{e}_{\lambda}^{*}$ defined by $\left\langle\mathfrak{e}_{\lambda}^{*} \mid \mathfrak{e}_{\mu}\right\rangle=\delta_{\lambda \mu}$ are given by the expansion

$$
\mathfrak{e}_{\lambda}^{*}=\sum_{\mu \in(n, k)} \frac{s_{\mu}(y(\lambda))}{\left\|\mathfrak{e}_{\lambda}\right\|^{2}}\langle\mu|
$$

Proof. This is a direct consequence of our previous discussion, applying $\mathcal{P} \mathcal{T}$ to (4.3) and then using part (i) of Theorem 4.1 .

Remark 4.7. In [24] the operator $\hat{H}_{N}=-q \prod_{j=1}^{N} \sigma_{j}^{z}$ was defined, which differs from $H_{N}=q|0\rangle\langle 0|$, which simply is the projection onto the unique basis vector in $V_{0, q}$. 
In loc. cit. the decomposition $V_{q}^{\otimes N}=\bigoplus_{n=0}^{N} V_{n, q}$ has been employed to define via the eigenbasis $\left\{\mathfrak{e}_{\lambda}\right\}_{\lambda \in(n, k)}$ the following set of operators

$$
\hat{H}_{r} \mid V_{n, q}=\sum_{\lambda \in(n, k)} h_{r}(y(\lambda)) \frac{\mathfrak{e}_{\lambda} \otimes \mathfrak{e}_{\lambda}^{*}}{\left\|\mathfrak{e}_{\lambda}\right\|^{2}}, r \geq 0 .
$$

Clearly, we have $H_{r}=\hat{H}_{r}$ for $0 \leq r<N$. The new insight here is that these operators originate from a Yang-Baxter algebra which has a local description in terms of a statistical vertex model on a square lattice.

\subsection{THE VERLINDE ALGEBRA}

Since the $q$-dependence can be removed via a simple rescaling of the Bethe roots, $y \rightarrow q^{-\frac{1}{N}} y$ (compare with (4.8)) we now set for simplicity $q=1$. Interpret the eigenbasis $\left\{\mathfrak{e}_{\lambda}\right\}_{\lambda \in(n, k)}$ as a complete set of orthogonal idempotents of an associative, unital and commutative algebra. Next, we show that the resulting generalised matrix algebra is isomorphic to the Verlinde algebra $\mathcal{F}_{n, k}^{\mathbb{C}}$, where we recall from the introduction that $\mathcal{F}_{n, k} \cong q H^{*}\left(\mathrm{Gr}_{n, n+k}\right) /\langle q-1\rangle$.

THEOREM 4.8 (idempotents of the Verlinde algebra). Endow $V_{n}$ with the following product, $\mathfrak{e}_{\lambda} \star \mathfrak{e}_{\mu}:=\delta_{\lambda \mu}\left\|\mathfrak{e}_{\lambda}\right\|^{2} \mathfrak{e}_{\lambda}$ and bilinear form $\eta(|\lambda\rangle,|\mu\rangle)=\delta_{\lambda \vee} \mu$. Then

(1) $\left(V_{n}, \star, \eta\right)$ is a commutative Frobenius algebra.

(2) The map $|\lambda\rangle \mapsto s_{\lambda}$ is an algebra isomorphism $\left(V_{n}, \star\right) \cong \mathcal{F}_{n, k}^{\mathbb{C}}$.

Remark 4.9. An analogous statement holds true for the $\widehat{\operatorname{su}}(n)_{k}$-WZNW fusion ring using so-called $\infty$-friendly walkers; see [24]. In [26, Section 5] the role of the Bethe vectors as idempotents has been highlighted and Section 7 of loc. cit. explains how the construction might generalise to other integrable models.

Proof. We compute the product expansion in the basis $\{|\lambda\rangle\}$. Exploiting (4.1), we find that

$$
\begin{aligned}
|\lambda\rangle \star|\mu\rangle & =\sum_{\alpha, \beta \in(n, k)}\left\langle\mathfrak{e}_{\alpha}^{*} \mid \lambda\right\rangle\left\langle\mathfrak{e}_{\beta}^{*} \mid \mu\right\rangle \mathfrak{e}_{\alpha} \star \mathfrak{e}_{\beta} \\
& =\sum_{\alpha \in(n, k)} \frac{s_{\lambda}(y(\alpha)) s_{\mu}(y(\alpha))}{\left\|\mathfrak{e}_{\alpha}\right\|^{2}} \mathfrak{e}_{\alpha}=\sum_{\nu \in(n, k)} C_{\lambda \mu}^{\nu, d}|\nu\rangle,
\end{aligned}
$$

where in the last step we have used, again, the definition (4.1) and the BertramVafa-Intriligator formula for Gromov-Witten invariants (see, e.g. [34, Cor 6.2, Eqn (6.1)]),

$$
C_{\lambda \mu}^{\nu, d}=\sum_{\alpha \in(n, k)} \frac{s_{\lambda}(y(\alpha)) s_{\mu}(y(\alpha)) s_{\nu}\left(y(\alpha)^{-1}\right)}{\left\|\mathfrak{e}_{\alpha}\right\|^{2}} .
$$


From this equality it is obvious that $|\emptyset\rangle$ is the identity with respect to the product $\star$ and using (4.1), we find

$$
|\emptyset\rangle=\sum_{\alpha \in(n, k)} \hat{\mathfrak{e}}_{\alpha}, \quad \hat{\mathfrak{e}}_{\alpha}=\mathfrak{e}_{\alpha} /\left\|\mathfrak{e}_{\alpha}\right\|^{2} .
$$

We now turn to the Frobenius structure. That $\eta$ is non-degenerate follows from the observation that $|\lambda\rangle \mapsto\left|\lambda^{\vee}\right\rangle$ simply permutes the basis elements in $V_{n}$. Compatibility of $\eta$ with the product amounts to the identity

$$
\eta(|\lambda\rangle \star|\mu\rangle,|\nu\rangle)=C_{\lambda \mu \nu}=C_{\mu \nu \lambda}=\eta(|\lambda\rangle,|\mu\rangle \star|\nu\rangle),
$$

where $C_{\lambda \mu \nu}:=C_{\lambda \mu}^{\nu^{\vee}, d}$ and we have used the known $S_{3}$-invariance of Gromov-Witten invariants, $C_{\lambda \mu \nu}=C_{\pi(\lambda) \pi(\mu) \pi(v)}$ for all $\pi \in S_{3}$, which is immediate from their geometric definition; see, e.g. [5].

Our main motivation to emphasise the Frobenius structure is the connection with the toric Schur polynomials mentioned after Proposition 1.1 in the introduction.

Proof of Proposition 1.1. Let $\mathfrak{m}: \mathcal{F}_{n, k} \otimes \mathcal{F}_{n, k} \rightarrow \mathcal{F}_{n, k}$ be the regular representation or multiplication map, $\mathfrak{m}\left(s_{\mu} \otimes s_{\nu}\right)=s_{\mu} \star s_{\nu}$, and $\mathfrak{m}^{*}: \mathcal{F}_{n, k}^{*} \rightarrow \mathcal{F}_{n, k}^{*} \otimes \mathcal{F}_{n, k}^{*}$ its dual map with the Frobenius isomorphism $\Phi: \mathcal{F}_{n, k} \rightarrow \mathcal{F}_{n, k}^{*}$ given by $\Phi: s_{\lambda} \mapsto \eta\left(s_{\lambda}, \bullet\right)$. Then, we recall that the coproduct $\Delta_{n, k}$ is obtained via the following commutative diagram,

$$
\begin{aligned}
& \mathcal{F}_{n, k} \stackrel{\Delta_{n, k}}{\longrightarrow} \mathcal{F}_{n, k} \otimes \mathcal{F}_{n, k} \\
& \downarrow \Phi \quad\lfloor\Phi \otimes \Phi . \\
& \mathcal{F}_{n, k}^{*} \stackrel{\mathfrak{m}^{*}}{\longrightarrow} \mathcal{F}_{n, k}^{*} \otimes \mathcal{F}_{n, k}^{*}
\end{aligned}
$$

Thus, we compute $\mathfrak{m}^{*} \circ \Phi\left(s_{\lambda}\right)\left(s_{\mu} \otimes s_{\nu}\right)=\Phi\left(s_{\lambda}\right)\left(s_{\mu} \star s_{\nu}\right)=C_{\lambda \mu \nu}$. Assuming on the other hand that $\Delta_{n, k} s_{\lambda}=\sum_{d, \alpha} s_{\lambda / d / \alpha} \otimes s_{\alpha}$ with $s_{\lambda / d / \alpha}=\sum_{\beta} C_{\alpha \beta}^{\lambda, d} s_{\beta}$, we find

$$
(\Phi \otimes \Phi) \Delta_{n, k}\left(s_{\lambda}\right)\left(s_{\mu} \otimes s_{v}\right)=\sum_{d, \alpha, \beta} C_{\alpha \beta}^{\lambda, d}\left(\Phi\left(s_{\beta}\right) \otimes \Phi\left(s_{\alpha}\right)\right)\left(s_{\mu} \otimes s_{v}\right)=C_{\lambda^{\vee} \mu^{\vee} v^{\vee}} .
$$

Exploiting invariance under Poincaré duality (see, e.g. [5]), $C_{\lambda^{\vee} \mu^{\vee} v^{\vee}}=C_{\lambda \mu \nu}$, the assertion now follows.

\section{Bijections Between Walks and Toric Tableaux}

In this section, we prove that the lattice configurations of the vicious and osculating walker models are in bijection with toric tableaux which were used in $[5,33]$. This will provide a combinatorial link between these statistical mechanics models and the quantum cohomology ring: the partition functions of vicious and osculating walkers on the cylinder are toric Schur functions. 


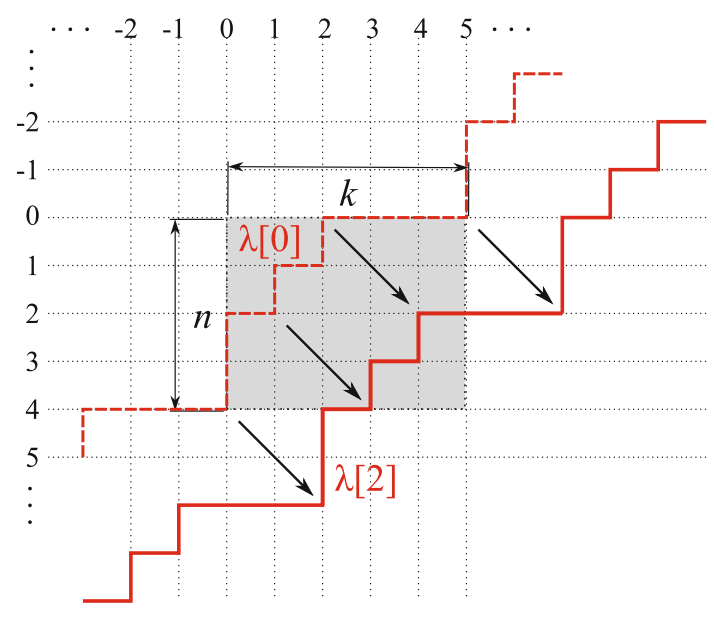

Figure 6. Example of a cylindric loop.

To keep this article self-contained we start by recalling the definition of toric tableaux following [33]. As mentioned earlier, toric tableaux are a particular subset of cylindric tableaux; see [18] for the original definition of cylindric (plane) partitions, lattice paths and cylindric Schur functions.

DEFINITION 5.1 (cylindric loops). Let $\lambda=\left(\lambda_{1}, \ldots, \lambda_{n}\right) \in(n, k)$ and define the following associated cylindric loops $\lambda[r]$ for any $r \in \mathbb{Z}$,

$$
\lambda[r]:=\left(\ldots, \lambda_{n}+\underset{r}{r}+\underset{r+1}{k} \lambda_{1}+r, \ldots, \underset{r+n}{\lambda_{n}+r} \underset{r+n+1}{\lambda_{1}+r-k}, \ldots\right) .
$$

For $r=0$, the cylindric loop can be visualised as a path in $\mathbb{Z} \times \mathbb{Z}$ determined by the outline of the Young diagram of $\lambda$ which is periodically continued with respect to the vector $(n,-k)$. For $r \neq 0$, this line is shifted $r$ times in the direction of the lattice vector $(1,1)$; see Figure 6 for an illustration.

DEFINITION 5.2 (cylindric skew diagrams). Given two partitions $\lambda, \mu \in(n, k)$ denote by $\lambda / d / \mu$ the set of squares between the two lines $\lambda[d]$ and $\mu[0]$ modulo integer shifts by $(n,-k)$,

$$
\lambda / d / \mu:=\left\{\langle i, j\rangle \in \mathbb{Z} \times \mathbb{Z} /(n,-k) \mathbb{Z} \mid \lambda[d]_{i} \geq j>\mu[0]_{i}\right\}
$$

We shall refer to $\lambda / d / \mu$ as a cylindric skew diagram of degree $d$.

A cylindric skew diagram $\nu / d / \mu$ which has at most one box in each column will be called a (cylindric) horizontal strip and one which has at most one box in each row a (cylindric) vertical strip. The length of such strips will be the number of boxes within the skew diagram. 


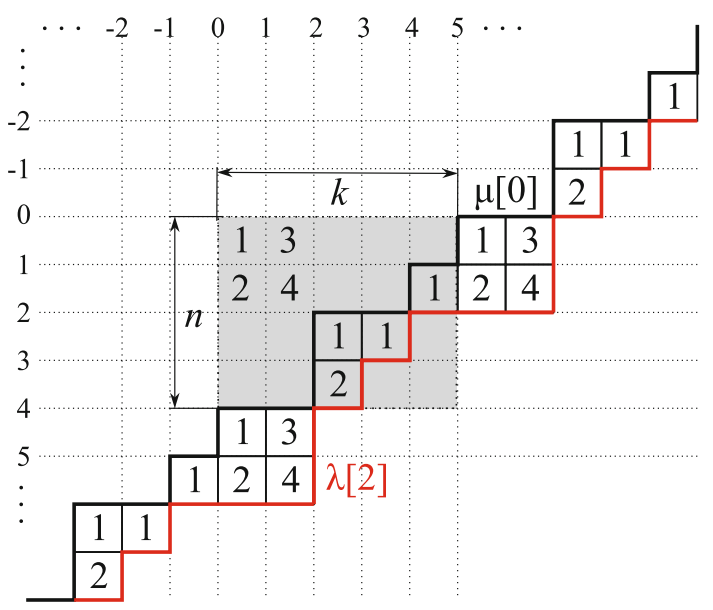

Figure 7. Example of a toric tableau. On the left it is indicated how the toric skew diagram is constructed from the cylindric loops of $\lambda$ and $\mu$. The right picture shows how the squares outside the bounding box are moved to the inside.

DEFINITION 5.3 (toric skew diagrams). A cylindric skew diagram is called toric if it has at most $k$ squares in each row.

See Figure 7 for an example of a toric skew diagram. Note that for $d=0$, we recover the familiar skew diagram of two partitions, i.e. $\lambda / 0 / \mu=\lambda / \mu$.

DEFINITION 5.4 (cylindric tableaux). A (semi-standard) tableau $T$ of cylindric shape $\lambda / d / \mu$ is a mapping $T: \lambda / d / \mu \mapsto \mathbb{N}$ of the squares of the associated diagram such that in each row and column of connected squares the numbers are, respectively, weakly increasing (left to right) and strictly increasing (top to bottom).

See Figure 7 for an example. As for ordinary skew diagrams, we define the weight vector $\alpha(T)=\left(\alpha_{1}, \alpha_{2}, \ldots\right)$ by setting $\alpha_{i}$ to be the number of $i$-entries in $T$.

DEFINITION 5.5 (Quantum Kostka numbers). The cardinality of the set of all cylindric tableaux $T$ of shape $\nu / d / \mu$ and weight $\alpha$ is denoted by $K_{v / d / \mu, \alpha}$.

As already pointed out in the introduction the quantum Kostka number $K_{v / d / \mu, \alpha}$ in (1.3) equals the number of semi-standard cylindric tableaux of weight $\alpha$, and specialises for $d=0$ to the ordinary Kostka number $K_{v / \mu, \alpha}$.

We will also make use of $K_{v^{\prime} / d / \mu^{\prime}, \alpha}$, the number of conjugate cylindric and toric diagrams $v^{\prime} / d / \mu^{\prime}$, which are obtained by interchanging $n$ and $k$. Here, $v^{\prime}, \mu^{\prime} \in(k, n)$ denote the conjugate partitions of $\nu, \mu$.

DEFINITION 5.6 (Cylindric Schur functions). Introduce the following generalisation of a skew Schur function, 


$$
s_{\nu / d / \mu}\left(x_{1}, x_{2}, \ldots\right):=\sum_{|T|=\nu / d / \mu} x^{T}=\sum_{\alpha} K_{\nu / d / \mu, \alpha} m_{\alpha}\left(x_{1}, x_{2}, \ldots\right)
$$

where $m_{\alpha}$ are the monomial symmetric functions in an infinite set of variables $x_{i}$ and the sum runs over all cylindric tableaux of fixed shape $v / d / \mu$.

Note that the cylindric Schur function (5.1) specialises to an ordinary skew Schur function for $d=0$.

DEFINITION 5.7 (Toric Schur functions). Specialising $x_{r}=0, r>n$ the cylindric Schur functions are called toric Schur functions, i.e. the sum in (5.1) runs only over toric tableaux.

We recall from [33, Lemma 5.2] that toric Schur functions are nonzero if and only if $\nu / d / \mu$ is a toric skew diagram.

\subsection{LATTICE CONFIGURATIONS AND QUANTUM KOSTKA NUMBERS}

Throughout this section, we assume $v, \mu \in(n, k)$. Denote by $\Gamma_{v, \mu}, \Gamma_{v, \mu}^{\prime}$ the sets of all allowed lattice configurations $\mathcal{C}, \mathcal{C}^{\prime}$ on the cylinder for the vicious and osculating walker models where the values of the lower and upper vertical lattice edges are fixed by the 01-words $w(v)$ and $w(\mu)$, respectively. It will also be convenient to consider the subsets $\Gamma_{v / d / \mu} \subset \Gamma_{\nu, \mu}$ of configurations which do have a fixed number of $2 d$ outer horizontal edges with value one (they come in pairs due to the quasiperiodic boundary conditions in the horizontal direction), including in particular the special case of $d=0(q=0)$ when there are no outer horizontal edges $\Gamma_{\nu / 0 / \mu}$. Finally, we introduce for each $\alpha=\left(\alpha_{1}, \ldots, \alpha_{n}\right) \in \mathbb{Z}_{\geq 0}^{n}$ the subsets

$$
\Gamma_{\nu / d / \mu}(\alpha):=\left\{\mathcal{C} \in \Gamma_{\nu / d / \mu}: \operatorname{wt}(\mathcal{C})=x^{\alpha}\right\}
$$

Analogously, we define for $\beta=\left(\beta_{1}, \ldots, \beta_{k}\right) \in \mathbb{Z}_{\geq 0}^{k}$ the set $\Gamma_{v / d / \mu}^{\prime}(\beta)$ as the lattice configurations $\mathcal{C}^{\prime}$ of the osculating walker model which have weight $\operatorname{wt}^{\prime}\left(\mathcal{C}^{\prime}\right)=x^{\beta}$.

PROPOSITION 5.8. The set of allowed lattice configurations $\Gamma_{\nu / d / \mu}$ and $\Gamma_{\nu / d / \mu}^{\prime}$ are in bijection with the sets of toric skew tableaux of shape $v / d / \mu$ and $\nu^{\prime} / d / \mu^{\prime}$, respectively. In particular,

$$
K_{v / d / \mu, \alpha}=\left|\Gamma_{v / d / \mu}(\alpha)\right|=\left|\Gamma_{v^{\prime} / d / \mu^{\prime}}^{\prime}(\alpha)\right|,
$$

where $\alpha=\left(\alpha_{1}, \ldots, \alpha_{n}\right)$ is some weight vector with non-negative integer entries.

Note that for $d=0$ and $\mu=\emptyset$ the statement specialises to Claim 4.3 which we used earlier to prove Lemma 4.2. 

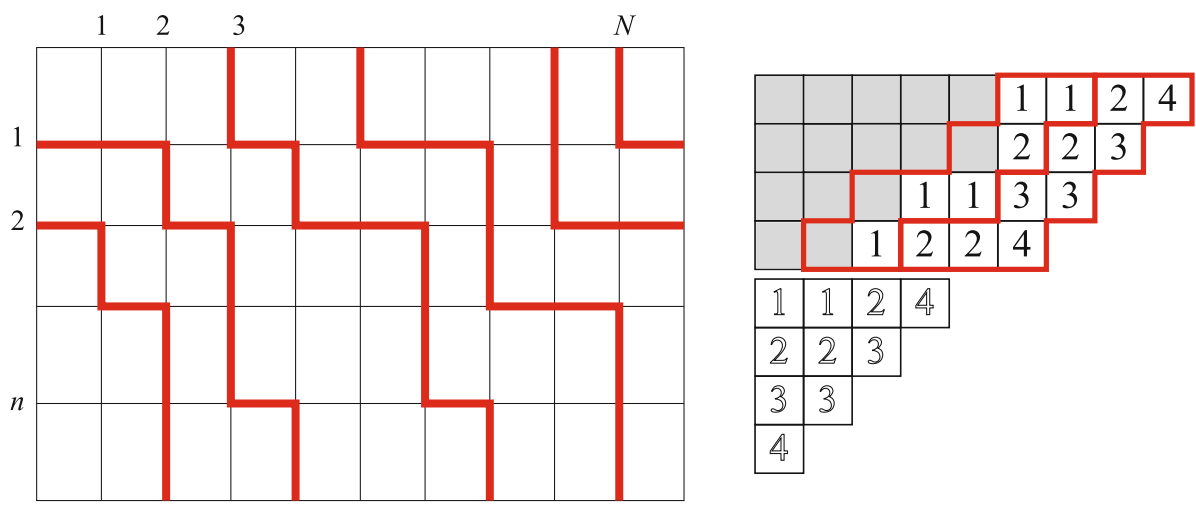

Figure 8. Example for constructing a toric tableau from the vicious walker configuration considered in the introduction with $N=9=n+k=4+5$ and $\lambda=(5,5,3,2)=\mu, v=(5,4,2,1)$. The shaded boxes on the right show the Young diagram of $\mu$ and removing from the toric skew diagram the two $(d=2)$ framed $N$-ribbons, one obtains the Young diagram of $v$. The boxes below belong to the periodic continuation of the tableau.

Proof. We concentrate on the vicious walker model, the generalisation to the osculating walker model will then be obvious. First, we state the bijection. Recall from Figure 3 that each lattice configuration $\mathcal{C} \in \Gamma_{\nu / d / \mu}$ defines an $n$-tuple of nonintersecting paths $\gamma=\left(\gamma_{1}, \ldots, \gamma_{n}\right)$ of which $d$ cross the boundary. Draw the Young diagram $Y(\mu)$ of $\mu$ in the bounding box $(n, k)$. Reading the 01-word of $\mu$ from left to right take the path which originates at $\ell_{1}(\mu)$, that is from the first 1-letter in $w(\mu)$, and note down the lattice rows of each of its horizontal edges starting from the top, say $i_{1} \leq \cdots \leq i_{r}$. Add a corresponding row of boxes with entries $i_{1} \leq \cdots \leq i_{r}$ to the bottom row of $Y(\mu)$. If the path has no horizontal edges do not add any boxes. Continue with the path originating from the second 1-letter in $w(\mu)$ at $\ell_{2}(\mu)$ and write the corresponding filled boxes in the row above the bottom row of $Y(\mu)$ starting at the first square which does not lie in $\mu$. Continue until you have reached the last 1-letter in $w(\mu)$; see Figure 8 for an example.

That the described map is indeed a bijection follows from the following lemma.

LEMMA 5.9. Each toric skew tableau $T$ of shape $\nu / d / \mu$ can be written as a sequence of toric horizontal strips, i.e. there is a unique sequence of cylindric loops $(\mu[0]=$ $\left.v^{(0)}\left[d_{0}\right], v^{(1)}\left[d_{1}\right], \ldots, v^{(n)}\left[d_{n}\right]=v[d]\right)$ such that $v^{(i+1)} /\left(d_{i+1}-d_{i}\right) / v^{(i)}$ is a horizontal strip and $\sum_{i} d_{i}=d$ with $d_{i}=0,1$.

Since toric tableaux can be seen as a special subset of ordinary (semi-standard) tableaux of shape $\nu[d] / \mu$ the proof of this lemma follows along very similar lines as in the case of ordinary tableaux (see, e.g. [29, Chap I]) and we therefore omit it.

Thus, it suffices to derive the assertion for $n=1$ in which case $d=0$ or 1 . Then we have the following generalisation of (2.2) to cylindric loops

$$
\mu[0]_{n+1-i}=\ell_{i}(\mu)-i \quad \text { and } \quad \lambda[1]_{n+1-i}=\ell_{i+1}(\lambda)-i,
$$



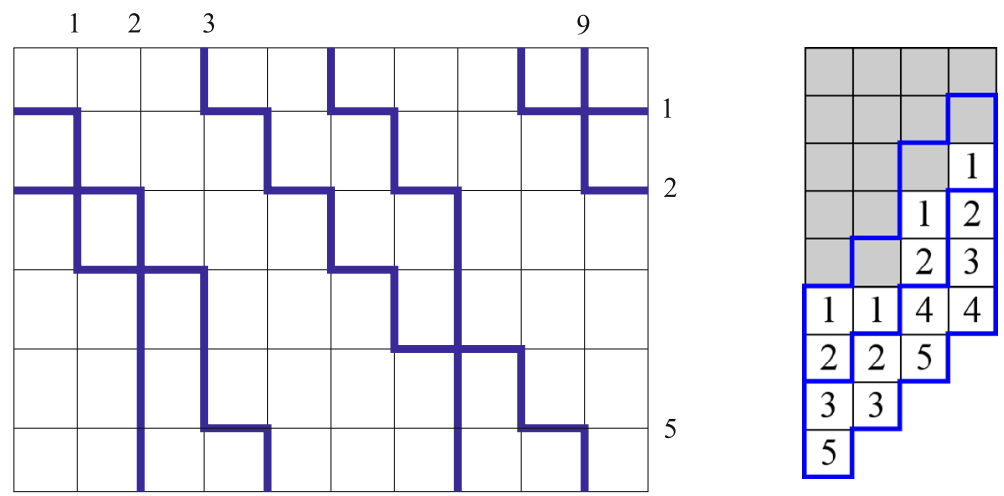

Figure 9. Example for constructing a conjugate toric tableau from the osculating walker configuration considered in the introduction. The shaded boxes on the right now show the Young diagram of $\mu^{\prime}$ and removing from the toric skew diagram the two $N$-ribbons $(d=2)$ framed in blue, one obtains the Young diagram $v^{\prime}$.

where $\ell_{i+n}(\mu)=\ell_{i}(\mu)+N, \ell_{i+n}(\lambda)=\ell_{i}(\lambda)+N$. Analogously, one finds for the conjugate loops,

$$
\mu[0]_{k+1-i}^{\prime}=\mu^{\prime}[0]_{k+1-i}=\ell_{i}\left(\mu^{\prime}\right)-i, \quad \lambda[1]_{k+1-i}^{\prime}=\lambda^{\prime}[1]_{k+1-i}=\ell_{i+1}\left(\lambda^{\prime}\right)-i
$$

with $\ell_{i+k}\left(\mu^{\prime}\right)=\ell_{i}\left(\mu^{\prime}\right)+N, \ell_{i+k}\left(\lambda^{\prime}\right)=\ell_{i}\left(\lambda^{\prime}\right)+N$. Using these formulae together with the action (3.15), (3.16), one now easily verifies that an allowed row configuration of the vicious walker model, i.e. a non-vanishing matrix element of a monomial in the hopping operators $f_{i}$ appearing in (3.21), defines a toric horizontal strip $v / d / \mu$ with $d=0,1$ and vice versa. In particular, the action of a consecutive string such as $f_{i} \cdots f_{2} f_{1} f_{N} \cdots f_{j} b_{\mu}=b_{\lambda}$ can only be nonzero if there are as many consecutive 0 -letters in $w(\mu)$ starting at $j$ and ending at $i$ as there are hopping operators $f_{l}$ in the string. Thus, the horizontal strip has at most length $k$.

The bijection for the osculating walkers is analogous. Start with the leftmost path originating at $\ell_{1}(\mu)$ and for each horizontal path edge in lattice row $i$ add a box labelled $i$ in the rightmost column of the $k \times n$ bounding box beneath the Young diagram of $\mu^{\prime}$. Continue with the second path placing the boxes now in the second column from the right and so forth. The result is a conjugate toric tableau of shape $v^{\prime} / d / \mu^{\prime}$; see Figure 9 for an example.

The proof that the described map is indeed a bijection employs the expansion (3.34) and follows closely along similar lines as in the previous case of vicious walkers. We therefore omit it.

Number the lattice columns of $\mathbb{L}\left(\mathbb{L}^{\prime}\right)$ from left to right. That is, the $i$ th column is the collection of horizontal lattice edges $\left(p, p^{\prime}\right) \in \mathbb{E}\left(\mathbb{E}^{\prime}\right)$ such that $p_{1}=i$ and $p_{1}^{\prime}=i+1$. Let $\mathcal{C}: \mathbb{E} \rightarrow\{0,1\}\left(\mathcal{C}^{\prime}: \mathbb{E}^{\prime} \rightarrow\{0,1\}\right)$ be a vicious (osculating) walker configuration with start and end positions $\ell(v)$ and $\ell(\mu), v, \mu \in(n, k)$. The number of horizontal path edges in column $i$ is the sum over the values of the horizontal lattice edges in column $i$ in configuration $\mathcal{C}\left(\mathcal{C}^{\prime}\right)$. 
LEMMA 5.10. The number of horizontal path edges in lattice column $i$ is

$$
\theta_{i}(\nu, \mu, d)=d+n_{i}(\mu)-n_{i}(\nu), \quad 1 \leq i \leq N .
$$

In particular, we have that $d \geq d_{\min }(\nu, \mu):=\max _{i \in I}\left\{n_{i}(\nu)-n_{i}(\mu)\right\}$.

Remark 5.11. The integer $d_{\min }\left(v^{\vee}, \mu\right)$ is the minimal power appearing in the quantum product $s_{\mu} * s_{v}$; see [16]. In fact, there exists an interval of integers $\left[d_{\min }, d_{\max }\right]$ describing all powers occurring in this product. This was first conjectured in [39] and proved in [33].

Proof. Denote by $w_{i}^{(j)}, v_{i}^{(j)} \in\{0,1)$ the values of the, respectively, horizontal and vertical lattice edges in column $i$ and row $j$ in the configuration $\mathcal{C}$ or $\mathcal{C}^{\prime}$. Since the argument is completely analogous for both, vicious and osculating walkers, we only consider the former for the rest of the proof. By construction, we have $v_{i}^{(0)}=w_{i}(\mu)$ and $v_{i}^{(n)}=w_{i}(v)$. Note that the allowed configurations shown in Figure 3 preserve the sum of values on the $\mathrm{N}$ and $\mathrm{W}$ edge and the $\mathrm{E}$ and $\mathrm{S}$ edge, that is $w_{i-1}^{(j)}+v_{i}^{(j-1)}=v_{i}^{(j)}+w_{i}^{(j)}$. Hence, we compute

$$
\begin{aligned}
\theta_{i} & =w_{i}^{(1)}+w_{i}^{(2)}+\cdots+w_{i}^{(n)} \\
& =v_{i}^{(0)}+w_{i-1}^{(1)}-v_{i}^{(1)}+v_{i}^{(1)}+w_{i-1}^{(2)}-v_{i}^{(2)}+\cdots+v_{i}^{(n-1)}+w_{i-1}^{(n)}-v_{i}^{(n)} \\
& =\theta_{i-1}+v_{i}^{(0)}-v_{i}^{(n)}=\theta_{i-1}+w_{i}(\mu)-w_{i}(v) .
\end{aligned}
$$

The assertion now follows by observing that $\theta_{N}=d$, the number of horizontal path edges on the boundary.

LEMMA 5.12 Let $\lambda \in(n, k) . K_{\nu / d / \mu, \lambda}=0$ unless $|\lambda|+|\mu|-|\nu|=d N$.

Proof. Since $\theta_{N}(v, \mu, d)=d$ and the vicious and osculating paths are nonintersecting we must have that the paths starting at $\ell_{N+1-d}(\mu), \ldots, \ell_{N}(\mu)$ end at $\ell_{1}(v), \ldots, \ell_{d}(v)$, respectively. Thus, the paths starting at $\ell_{1}(\mu), \ldots, \ell_{N-d}(\mu)$ end at $\ell_{d+1}(v), \ldots, \ell_{N}(v)$.

Therefore, $|\lambda|=\sum_{i=1}^{n}\left(\ell_{i+d}(\nu)-\ell_{i}(\mu)\right)=|\nu|-|\mu|+d N$.

\subsection{PATH COUNTING AND GROMOV-WITTEN INVARIANTS}

We now compute the weighted sums over lattice configurations of the vicious and osculating walker models; these are called partition functions. Consider the following operator products $H\left(x_{1}\right) \cdots H\left(x_{n}\right) \in \mathbb{Z}\left[x_{1}, \ldots, x_{n}\right] \otimes \operatorname{End}\left(V_{q}^{\otimes N}\right)$ and $E\left(x_{1}\right) \cdots$ $E\left(x_{k}\right) \in \mathbb{Z}\left[x_{1}, \ldots, x_{k}\right] \otimes \operatorname{End}\left(V_{q}^{\otimes N}\right)$. Then by construction, we have for the vicious walker model

$$
\left\langle v\left|H\left(x_{1}\right) \cdots H\left(x_{n}\right)\right| \mu\right\rangle=\sum_{\mathcal{C} \in \Gamma_{v, \mu}} \prod_{\langle i, j\rangle \in \mathbb{L}} \operatorname{wt}\left(\mathrm{v}_{i, j}\right) \in \mathbb{Z}\left[x_{1}, \ldots, x_{n}\right],
$$


and for the osculating walker model,

$$
\left\langle\nu\left|E\left(x_{1}\right) \cdots E\left(x_{k}\right)\right| \mu\right\rangle=\sum_{\mathcal{C}^{\prime} \in \Gamma_{v, \mu}^{\prime}} \prod_{\langle i, j\rangle \in \mathbb{L}^{\prime}} \mathrm{wt}^{\prime}\left(\mathrm{v}_{i, j}\right) \in \mathbb{Z}\left[x_{1}, \ldots, x_{k}\right],
$$

where $\langle v|X| \mu\rangle$ is shorthand for $\langle b(v), X b(\mu)\rangle$ and $\mathrm{wt}\left(\mathrm{v}_{i, j}\right), \mathrm{wt}^{\prime}\left(\mathrm{v}_{i, j}\right)$ denote the vertex weights of the vicious and osculating walker models in Figures 3, 4.

COROLLARY 5.13 The partition function (5.5) has the following expansion in toric Schur polynomials

$$
\left\langle\nu\left|H\left(x_{1}\right) \cdots H\left(x_{n}\right)\right| \mu\right\rangle=\sum_{d \geq 0} q^{d} s_{v / d / \mu}\left(x_{1}, \ldots, x_{n}\right) .
$$

The analogous expansion of the partition function (5.6) for the osculating walker model reads

$$
\left\langle v\left|E\left(x_{1}\right) \cdots E\left(x_{k}\right)\right| \mu\right\rangle=\sum_{d \geq 0} q^{d} s_{v^{\prime} / d / \mu^{\prime}}\left(x_{1}, \ldots, x_{k}\right) .
$$

In particular, for $\lambda \in(n, k)$ the quantum Kostka numbers are given by the matrix elements

$$
q^{d} K_{v / d / \mu, \lambda}=\left\langle v\left|H_{\lambda}\right| \mu\right\rangle=\left\langle v^{\prime}\left|E_{\lambda}\right| \mu^{\prime}\right\rangle
$$

where $H_{\lambda}=H_{\lambda_{1}} \cdots H_{\lambda_{n}}, E_{\lambda}=E_{\lambda_{1}} \cdots E_{\lambda_{n}}$ and $d=(|\lambda|+|\mu|-|\nu|) / N$.

Proof. Let $m_{\lambda}$ be the monomial symmetric function. We have the identities

$$
\begin{aligned}
\left\langle\nu\left|H\left(x_{1}\right) \cdots H\left(x_{n}\right)\right| \mu\right\rangle & =\sum_{\lambda \in(n, k)}\left\langle\nu\left|H_{\lambda}\right| \mu\right\rangle m_{\lambda}\left(x_{1}, \ldots, x_{n}\right) \\
& =\sum_{\lambda \in(n, k)} q^{d(\lambda)} K_{v / d / \mu, \lambda} m_{\lambda}\left(x_{1}, \ldots, x_{n}\right),
\end{aligned}
$$

where (5.10) is a direct consequence of the expansion (3.21) and the fact that the partition function (5.5) must be symmetric in the $x_{i}$ 's due to $H(x) H(y)=$ $H(y) H(x)$. The asserted identity (5.11) with $d(\lambda) N=|\lambda|+|\mu|-|\nu|$ follows from the bijection described in the proof of Proposition 5.8 and Lemma 5.12. Recalling that the monomial symmetric functions form a basis in the ring of symmetric functions the last equality is proved. The argument is completely analogous for the osculating walkers.

We now recover the following result from [33, Thm 5.3] and [24, Thm 10.8].

COROLLARY 5.14. Let $n \neq 0, N$. One has the following expansion of toric Schur functions, 


$$
s_{\nu / d / \mu}\left(x_{1}, \ldots, x_{n}\right)=\sum_{|\lambda|+|\mu|-|\nu|=d N} C_{\lambda \mu}^{v, d} s_{\lambda}\left(x_{1}, \ldots, x_{n}\right),
$$

where $C_{\lambda \mu}^{\nu, d}=\left\langle\nu\left|S_{\lambda}\right| \mu\right\rangle$ and $S_{\lambda}=\operatorname{det}\left(H_{\lambda_{i}-i+j}\right)_{1 \leq i, j \leq n}$.

Proof. Recall from the ring of symmetric functions the familiar expansion $m_{\alpha}=$ $\sum_{\lambda} L_{\alpha \lambda} s_{\lambda}$, where $L_{\alpha \lambda}=\sum_{w}^{\prime}(-1)^{w}$ with the sum ranging over all permutations $w \in$ $S_{n}$ such that $w(\lambda+\rho)-\rho \in S_{n} \alpha$. Here $\rho=(n-1, \ldots, 2,1,0)$ is the Weyl vector. Employing this identity together with $S_{\lambda}=\sum_{w}(-1)^{w} H_{w(\lambda+\rho)-\rho}$, one proves that the expansion coefficients of the partition function (5.7) into Schur functions are the matrix elements $\left\langle v\left|S_{\lambda}\right| \mu\right\rangle$. That the latter equal the Gromov-Witten invariants can be derived from Cor 3.24 and is a consequence of (4.13), (4.14) together with the eigenvalue equations (4.6), (4.7) and Theorem 4.1.

Let $c(s)=j-i$ be the content and $h(s)=\lambda_{i}+\lambda_{j}^{\prime}-i-j+1$ the hook-length of a square $s=(i, j) \in \lambda$. As a special case of the last corollary we obtain the following solution to the counting problem of non-intersecting paths on the cylinder which is a refinement of the one stated in the introduction.

COROLLARY 5.15 Setting $x_{1}=\cdots=x_{n}=1$ we have that

$$
\left|\Gamma_{\nu / d / \mu}\right|=\sum_{\substack{\lambda \in(n, k) \\|\lambda|+|\mu|-|\nu|=d N}} C_{\lambda \mu}^{\nu, d} \prod_{s \in \lambda} \frac{n+c(s)}{h(s)}=\left|\Gamma_{\nu^{\prime} / d / \mu^{\prime}}^{\prime}\right| .
$$

Proof. Trivial consequence of the known identity $s_{\lambda}(1, \ldots, 1)=\prod_{s \in \lambda} \frac{n+c(s)}{h(s)}$ for Schur functions, see, e.g. [29, Chapter I, Ex 1(a), page 26]. The above result then simply states that setting $q=1$, we have

$$
\left|\Gamma_{v, \mu}\right|=\left\langle v\left|H^{n}\right| \mu\right\rangle=\sum_{d \geq 0} s_{v / d / \mu}(1, \ldots, 1)=\left\langle v^{\prime}\left|E^{k}\right| \mu^{\prime}\right\rangle=\left|\Gamma_{v^{\prime}, \mu^{\prime}}^{\prime}\right|
$$

where $H=H(1)$ and $E=E(1)$.

Remark 5.16 For $q=0$, we recover the result for configurations on the finite open strip by applying the Gessel-Viennot Theorem; see, e.g. [9,14,15,20-22]. This yields the familiar determinant (Jacobi-Trudi) formula for skew Schur functions. For general $q$ the only known expressions involve sums of determinants; see [31, Equation (6.4), page 299] and [18, Prop 1].

\section{Toric Schur Function Identities}

We are now in the position to derive the formula (1.16) and the generating function (1.15) stated in the introduction. We start with proving the identity (1.14). 
Proof of Proposition 1.5. It follows from our previous results (5.7) and (4.3) that we have the following expansion,

$$
\left\langle\lambda\left|H\left(y_{n}\right) \cdots H\left(y_{1}\right) B\left(x_{n}\right) \cdots B\left(x_{1}\right)\right| 0\right\rangle=x^{\delta_{n}} \sum_{\substack{\mu \in(n, k) \\ d \geq 0}} q^{d} s_{\lambda / d / \mu}(y) s_{\mu}(x) .
$$

But according to Proposition 1.1 the right hand side-apart from the monomial factor $x^{\delta_{n}}$-is the image of the Schubert class $s_{\lambda}$ under the coproduct of the Verlinde algebra. The analogous result holds true for osculating walkers using Lemma 3.19 .

We now invoke the commutation relations of the Yang-Baxter algebra (3.18) to provide a closed formula for the above matrix element in terms of the divided difference operators (3.11).

Proof of Corollary 1.6. Recall Lemma 3.5, then it follows from the definition $H(x ; q)=A(x)+q D(x)$ that $H\left(x_{i+1} ; q\right) B\left(x_{i}\right) f=\partial_{i} B\left(x_{i+1}\right) H\left(x_{i} ;-q\right) f$ for any $f \in$ $\mathbb{C}\left[x_{i}, x_{i+1}, q\right] \otimes V^{\otimes N}$ which is symmetric in $x_{i}, x_{i+1}$. For our purposes it suffices to make the stronger assumption that $f$ does not depend on $x_{i}, x_{i+1}$ and, thus, we simply write $H\left(x_{i+1} ; q\right) B\left(x_{i}\right)=\partial_{i} B\left(x_{i+1}\right) H\left(x_{i} ;-q\right)$ as an operator identity. We prove the following formula by induction,

$$
H\left(x_{n} ; q\right) B\left(x_{n-1}\right) \cdots B\left(x_{1}\right)=\partial_{n-1} \cdots \partial_{2} \partial_{1} B\left(x_{n}\right) \cdots B\left(x_{2}\right) H\left(x_{1} ;(-1)^{n-1} q\right) .
$$

As just discussed Lemma 3.5 tells us that the assertion is true for $n=2$ by setting $i=1$. Assume it holds true for some $n \geq 2$. Then it follows from Lemma 3.5 that

$$
\begin{aligned}
H & \left(x_{n+1} ; q\right) B\left(x_{n}\right) B\left(x_{n-1}\right) \cdots B\left(x_{1}\right) \\
& =\partial_{n} B\left(x_{n+1}\right) H\left(x_{n} ;-q\right) B\left(x_{n-1}\right) \cdots B\left(x_{1}\right) \\
& =\partial_{n} \partial_{n-1} \cdots \partial_{1} B\left(x_{n}\right) B\left(x_{n-1}\right) \cdots B\left(x_{2}\right) H\left(x_{1} ;(-1)^{n} q\right)
\end{aligned}
$$

which completes the proof by induction.

Setting $y_{i}=x_{i+n}$ and $\nabla_{i}=\partial_{n-1+i} \cdots \partial_{i+1} \partial_{i}$ one now computes

$$
\begin{aligned}
H & \left(y_{n} ; q\right) \cdots H\left(y_{1} ; q\right) B\left(x_{n}\right) \cdots B\left(x_{1}\right) \\
& =H\left(x_{2 n} ; q\right) \cdots H\left(x_{n+2} ; q\right) \nabla_{1} B\left(x_{n+1}\right) \cdots B\left(x_{2}\right) H\left(x_{1} ;(-1)^{n} q\right) \\
& =\nabla_{1} H\left(x_{2 n} ; q\right) \cdots H\left(x_{n+2} ; q\right) B\left(x_{n+1}\right) \cdots B\left(x_{2}\right) H\left(x_{1} ;(-1)^{n} q\right) \\
& =\cdots \\
& =\nabla_{1} \cdots \nabla_{n} B\left(x_{2 n}\right) \cdots B\left(x_{n+1}\right) H\left(x_{n} ;(-1)^{n} q\right) \cdots H\left(x_{1} ;(-1)^{n} q\right) .
\end{aligned}
$$

Taking the matrix element $\langle\lambda|\ldots| 0\rangle$ on both sides of the last identity and exploiting that $H\left(x_{i} ;(-1)^{n} q\right)|0\rangle=\left(1+q x_{i}^{N}\right)|0\rangle$ we find the asserted generating function (1.15) and the identity (1.16). 
EXAMPLE 6.1. Consider $q H^{*}\left(\mathrm{Gr}_{2,4}\right)$. There are six basis elements each corresponding to a partition in the $2 \times 2$ bounding box. Pick any $\lambda$ with Young diagram inside the $2 \times 2$ bounding box. Invoking (1.15) and (1.16) one first computes $x^{-\delta_{2}} D_{1} D_{2} \cdots D_{n} y^{\delta_{2}} F_{\lambda}(x ; y)$ via (3.11) before expanding the result into Schur functions in the $x$-variables,

$$
\begin{array}{c||l|c|c|c|}
\lambda & \emptyset|(1,0)|(1,1) \mid(2,0) & (2,1) & (2,2) \mid \\
\hline s_{\lambda} & \left.1\left|x_{1}+x_{2}\right| x_{1} x_{2}\right|_{1} ^{2}+x_{1} x_{2}+x_{2}^{2}\left|x_{1}^{2} x_{2}+x_{1} x_{2}^{2}\right| x_{1}^{2} x_{2}^{2} \mid
\end{array} .
$$

Since the latter form a basis this amounts to solving a linear system of equations. These steps can be readily implemented on a computer using one's favourite symbolic computation package such as Mathematica or Maple. The solution yields the toric Schur functions in the $y$-variables on the left hand side of (1.16) which in a similar manner can be expanded into Schur functions. The table below lists the expansion of the toric Schur functions $\sum_{d \geq 0} q^{d} s_{\lambda / d / \mu}(y)$ for the given values of $\lambda$ and $\mu$.

\begin{tabular}{||c||c|c|c|c|c|}
\hline$\lambda \backslash \mu$ & $(1,0)$ & $(1,1)$ & $(2,0)$ & $(2,1)$ & $(2,2)$ \\
\hline$\emptyset$ & $q s_{2,1}$ & $q s_{2,0}$ & $q s_{1,1}$ & $q s_{1,0}$ & $q^{2} s_{2,2}$ \\
\hline$(1,0)$ & $1+q s_{2,2}$ & $q s_{2,1}$ & $q s_{2,1}$ & $q s_{2,0}+q s_{1,1}$ & $q s_{1,0}$ \\
\hline$(1,1)$ & $s_{1,0}$ & 1 & $q s_{2,2}$ & $q s_{2,1}$ & $q s_{2,0}$ \\
\hline$(2,0)$ & $s_{1,0}$ & $q s_{2,2}$ & 1 & $q s_{2,1}$ & $q s_{1,1}$ \\
\hline$(2,1)$ & $s_{2,0}+s_{1,1}$ & $s_{1,0}$ & $s_{1,0}$ & $1+q s_{2,2}$ & $q s_{2,1}$ \\
\hline$(2,2)$ & $s_{2,1}$ & $s_{1,1}$ & $s_{2,0}$ & $s_{1,0}$ & 1 \\
\hline
\end{tabular}

Since $s_{\lambda / d / \mu}=\sum_{\nu \in(n, k)} C_{\mu \nu}^{\lambda, d} s_{\nu}$ one can easily read off the Gromov-Witten invariants and the above table contains the complete multiplication table for $q H^{*}\left(\mathrm{Gr}_{2,4}\right)$. For instance, the table entry $\lambda=(1,0)$ and $\mu=(2,1)$ reads $q s_{2,0}+q s_{1,1}$ from which we infer $C_{(2,1),(2,0)}^{(1,0),}=1$ and $C_{(2,1),(1,1)}^{(1,0) 1}=1$. For more complicated examples with $n+k>4$ Gromov-Witten invariants greater than one will occur.

COROLLARY 6.2. Assume $N=n+k$ with $k \geq n$ and $0<r \leq n$. For any $\lambda, \mu \in(n, k)$ we have the following identities for toric Schur functions

$$
\begin{aligned}
& \sum_{\substack{d+d^{\prime}=r \\
\mu \in(n, k)}} s_{\lambda / d / \mu}\left(x_{1}, \ldots, x_{n}\right) s_{\mu^{\prime} / d^{\prime} / v^{\prime}}\left(-x_{1}, \ldots,-x_{n}, x_{n+1}, \ldots, x_{k}\right) \\
& \quad=\sum_{d=0}^{r}(-1)^{n(r-d)} e_{r-d}\left(x_{1}^{N}, \ldots, x_{n}^{N}\right) s_{\lambda^{\prime} / d^{\prime} / v^{\prime}}\left(x_{n+1}, \ldots, x_{k}, 0, \ldots, 0\right)
\end{aligned}
$$

which for $n=k$ specialise to the statement in the introduction. 
Proof. Consider the matrix element $\left\langle\lambda\left|H\left(x_{1}\right) \cdots H\left(x_{n}\right) E\left(y_{1}\right) \cdots E\left(y_{k}\right)\right| v\right\rangle$. Then according to (5.7) and (5.8), we have

$$
\left\langle\lambda\left|H\left(x_{1}\right) \cdots H\left(x_{n}\right) E\left(y_{1}\right) \cdots E\left(y_{k}\right)\right| v\right\rangle=\sum_{\substack{\mu \in(n, k) \\ d, d^{\prime} \geq 0}} q^{d+d^{\prime}} s_{\lambda / d / \mu}(x) s_{\mu^{\prime} / d^{\prime} / \nu^{\prime}}(y) .
$$

On the other hand setting $y_{i}=-x_{i}$ for $i=1,2, \ldots, n$ we obtain from the functional relation (3.40) that

$$
\left\langle\lambda\left|H\left(x_{1}\right) \cdots H\left(x_{n}\right) E\left(y_{1}\right) \cdots E\left(y_{k}\right)\right| v\right\rangle=\left\langle\lambda\left|E\left(y_{n+1}\right) \cdots E\left(y_{k}\right)\right| v\right\rangle \prod_{i=1}^{n}\left(1+(-1)^{n} q x_{i}^{N}\right) .
$$

Equating powers of $q$ on both sides, we find the claimed identities.

\section{Acknowledgements}

The research presented in this article has in part been financially supported by a University Research Fellowship of the Royal Society, by the Engineering and Physical Sciences Research Council grants EP/I037636/1 and GR/R93773/01 as well as the trimester programme Integrability in Geometry and Mathematical Physics (1 January - 30 April 2012) at the Hausdorff Research Institute for Mathematics, Bonn, Germany. Some of the results have been presented during the trimester workshop Integrability in Topological Field Theory (16-20 April 2012) organised by Albrecht Klemm and Katrin Wendland. The author wishes to thank the trimester organisers, Franz Pedit, Ulrich Pinkall, Iskander Taimanov, Alexander Veselov and Katrin Wendland and the staff of the Hausdorff Institute for their kind invitation and hospitality. It is a pleasure to thank Vassily Gorbounov and Catharina Stroppel for discussions.

Open Access This article is distributed under the terms of the Creative Commons Attribution License which permits any use, distribution, and reproduction in any medium, provided the original author(s) and the source are credited.

\section{References}

1. Agnihotri, S. Quantum Cohomology and the Verlinde Algebra. Ph.D. Thesis, University of Oxford (1995)

2. Agnihotri, S., Woodward, C.: Eigenvalues of products of unitary matrices and quantum Schubert calculus. Math. Res. Lett. 5, 817-836 (1998)

3. Baxter, R.J. Exactly Solved Models in Statistical Mechanics. Academic Press Inc, Harcourt Brace Jovanovich Publishers, London (1989, Reprint of the 1982 original)

4. Bertram, A.: Quantum Schubert calculus. Adv. Math. 128, 289-305 (1997)

5. Bertram, A., Ciocan-Fontanine, I., Fulton, W. Quantum multiplication of Schur polynomials. J. Algebra 219, 728-746 (1999)

6. Bogoliubov, N.M., Izergin, A.G., Korepin, V.E.: Quantum inverse scattering method and correlation functions. In: Cambridge Monographs on Mathematical Physics. Cambridge University Press, Cambridge (1993) 
7. Borodin, A.: Periodic Schur process and cylindric partitions. Duke Math. J. 140(3), 391-468 (2007)

8. Brak, R.: Osculating lattice paths and alternating sign matrices. In: Proceedings of Formal Power Series and Algebraic Combinatorics 9, 120-130 (1997)

9. Brak, R., Owczarek, A.: A combinatorial interpretation of the free-fermion condition of the six-vertex model. J. Phys. A Math. Gen. 32, 3497-3503 (1999)

10. Buch, A.S.: Quantum cohomology of Grassmannians. Compos. Math. 137(2), 227-235 (2003)

11. Buch, A.S., Kresch, A., Tamvakis, H.: Gromov-Witten invariants on Grassmannians. J. Am. Math. Soc. 16(4), 901-915 (electronic) (2003)

12. Fisher, M.E.: Walks, walls, wetting and melting. J. Stat. Phys. 34, 667-729 (1984)

13. Fomin, F.; Greene, C. Noncommutative Schur functions and their applications. Discrete Math. 193, 179-200 (1998). Selected papers in honor of Adriano Garsia (Taormina, 1994)

14. Forrester, P.: Exact solution of the lock step model of vicious walkers. J. Phys. A 23, 1259-73 (1990)

15. Forrester, P.: Exact results for vicious walker models of domain walls. J. Phys. A 24, 203-218 (1991)

16. Fulton, W., Woodward, C.: On the quantum product of Schubert classes. J. Algebraic Geom. 13, 641-661 (2004)

17. Gepner, D.: Fusion rings and geometry. Commun. Math. Phys. 141(2), 381-411 (1991)

18. Gessel, I.M., Krattenthaler, C.: Cylindric partitions. Trans. Am. Math. Soc. 349, 429479 (1997)

19. Gorbounov, V., Rimanyi, R., Tarasov, V., Varchenko, A.: Cohomology of the cotangent bundle of a flag variety as a Yangian Bethe algebra. arXiv preprint arXiv:1204.5138 (2012)

20. Guttmann, A., Owczarek, A., Viennot, X.: Vicious walkers and Young tableaux. I. Without walls. J. Phys. A 31(40), 8123-8135 (1998)

21. Krattenthaler, C., Guttmann, A., Viennot, X.: Vicious walkers, friendly walkers and Young tableaux II. With a wall. J. Phys. A 33, 8835-66 (2000)

22. Krattenthaler, C., Guttmann, A., Viennot, X. Vicious walkers, friendly walkers, and Young tableaux III. Between two walls. Special issue in honor of Michael E. Fisher's 70th birthday (Piscataway, NJ, 2001). J. Stat. Phys. 110(3-6), 1069-1086 (2003)

23. Intriligator, K.: Fusion residues. Modern Phys. Lett. A 6(38), 3543-3556 (1991)

24. Korff, C., Stroppel, C.: The $\widehat{\mathfrak{s l}}(n)_{k}$-WZNW fusion ring: a combinatorial construction and a realisation as quotient of quantum cohomology. Adv. Math. 225, 200-268 (2010)

25. Korff, C.: Noncommutative Schur polynomials and the crystal limit of the $U_{q} \widehat{\mathfrak{s l}}(2)-$ vertex model. J. Phys. A 43, 434021 (2010)

26. Korff, C.: The su(n) WZNW fusion ring as integrable model: a new algorithm to compute fusion coefficients. RIMS Kokyuroku Bessatsu B 28, 121-153 (2011)

27. Korff, C.: Cylindric Macdonald functions and a deformation of the Verlinde algebra. preprint; arxiv: 1110.6356

28. Lam, T.: Affine Stanley symmetric functions. Am. J. Math. 128, 1553-1586 (2006)

29. Macdonald, I.G.: Symmetric functions and Hall polynomials. In: Oxford Mathematical Monographs, 2nd edn. The Clarendon Press Oxford University Press, Clarendon (1995)

30. Maulik, D., Okounkov, A.: Quantum Groups and Quantum Cohomology, Preprint (2012), 1-276. arXiv preprint arXiv:1211.1287

31. McNamara, P.: Cylindric skew Schur functions. Adv. Math. 205, 275-312 (2006)

32. Nekrasov, N.A., Shatashvili, S.L.: Quantum integrability and supersymmetric vacua. In: Progress Theor. Phys. Suppl. 177, 105-119 (2009) 
33. Postnikov, A.: Affine approach to quantum Schubert calculus. Duke Math. J. 128, 473509 (2005)

34. Rietsch, K.: Quantum cohomology rings of Grassmannians and total positivity. Duke Math. J. 110(3), 523-553 (2001)

35. Rimanyi, R., Schechtman, V., Tarasov, V., Varchenko, A.: Cohomology of a flag variety as a Bethe algebra. Funct. Anal. Appl. 45(4), 252-264 (2011)

36. Siebert, B., Tian, G.: On quantum cohomology rings of Fano manifolds and a formula of Vafa and I ntriligator. Asian J. Math. 1(4), 679-695 (1997)

37. Vafa, C.: Topological mirrors and quantum rings. In: Essays on Mirror Manifolds, pp. 96-119. Int. Press, Hong Kong (1992)

38. Witten, E.: The Verlinde algebra and the cohomology of the Grassmannian. In: Geometry, Topology, \& Physics , Conf. Proc. Lecture Notes Geom. Topology, IV, pp. 357422. Int. Press, Cambridge (1995)

39. Yong, A.: Degree bounds in quantum Schubert calculus. Proc. Am. Math. Soc. 131, 2649-2655 (2003) 